\title{
Field Operators for Anyons and Plektons
}

\author{
Karl-Henning Rehren \\ II. Institut für Theoretische Physik, Universität Hamburg, Luruper Chaussee 149, W-2000 \\ Hamburg 50, Federal Republic of Germany
}

Received June 24, 1991

\begin{abstract}
Given its superselection sectors with non-abelian braid group statistics, we extend the algebra $\mathscr{A}$ of local observables into an algebra $\mathscr{F}$ containing localized intertwiner fields which carry the superselection charges. The construction of the inner degrees of freedom, as well as the study of their transformation properties (quantum symmetry), are entirely in terms of the superselection structure of the observables. As a novel and characteristic feature for braid group statistics, Clebsch-Gordan and commutation "coefficients" generically take values in the algebra $\mathscr{M}$ of symmetry operators, much as it is the case with quasi-Hopf symmetry. $\mathscr{A}, \mathscr{F}$, and $\mathscr{M}$ are all $C^{*}$ algebras, i.e. represented by bounded operators on a Hilbert space with positive metric.
\end{abstract}

\section{Introduction and Results}

It is very convenient and successful to describe quantum numbers of particles in terms of unobservable charged fields, which transform under a gauge group. This is motivated by the observation that non-trivial representations of the global gauge group give rise to superselection sectors for the gauge invariant quantities (observables), and is a posteriori justified by the result [1] of Doplicher and Roberts (referred to as the DR construction below), based on the theory of superselection sectors and statistics [2] of the same authors and Haag (DHR), that under reasonable assumptions in four space-time dimensions superselection sectors always arise by this mechanism, i.e. a gauge covariant field algebra can be constructed from the subalgebra of local observables and its superselection structure, such that its charged fields indeed generate the sectors. Essential for this construction is the fact that the superselection structure (i.e. multiplicities of irreducible subrepresentations $\pi_{\gamma}$ in a product of sectors $\pi_{\alpha} \times \pi_{\beta}$ ) coincides with the unitary representation theory of some compact group $G$ (i.e. multiplicities of $\mathscr{D}^{\gamma}$ within $\mathscr{D}^{\alpha} \otimes \mathscr{D}^{\beta}$ ).

Now, in recent analysis of conformal quantum field theory, models were discovered where this coincidence fails, and this failure has been traced back to a structural difference in the theory of superselection sectors for low-dimensional quantum physics in general. It may as well occur in $2+1$ dimensional theories with particle-like excitations carrying a "string of glue" with them. The new issue 
is the typical occurrence of braid group statistics [3] instead of familiar permutation group statistics. The "abelian" case has actually been discussed for a long time under the name of anyons or fractional statistics, and is reasonably well understood in rather conservative terms (path integrals or Wightman fields). In contrast, the "non-abelian" case (its particle-like excitations are called plektons) is still a challenge, mainly since a satisfactory understanding of the associated "gauge" symmetry is lacking. An intriguing observation from $2 d$ conformal models is, however, that the superselection structure of the chiral algebra matches (part of) the representation theory of a "quantum group" [4], raising much hope that the latter provide the new concept of symmetry. Unfortunately, attempts failed to define a field algebra with quantum group symmetry decently represented on a Hilbert space with positive metric, although recent progress [5] suggests that quasi-Hopf algebras might well work out.

Whether quantum groups are relevant in physics or not, their study taught us to keep an open mind towards what we mean by a "product" of representations of an algebra. We emphasize that for general algebras there is no such thing at all, and the DHR product $\pi_{\alpha} \times \pi_{\beta}$ mentioned above of representations (superselection sectors) of the algebra of local observables was a major progress of special physical relevance. For groups and Lie algebras, the natural composition is the tensor product, but yet this is not always the physically relevant one. E.g., for the Poincaré group the two-particle representation (in Fock space) differs from the tensor product of one-particle representations by the appropriate symmetrization prescription. For space-time covariance groups in low dimensions, the effect of the statistics on the composition of representations is even more drastic, allowing for non-additivity $(\bmod 1)$ of the relevant "spin" quantum number [6]. Indeed, the miraculous sum rules for the conformal spin $h$ in Wess-Zumino-Witten and minimal models and the related monodromy problem stood at the beginning of the study of non-abelian braid group statistics $[7,8]$.

As for "inner" symmetries, their structure must not even be that of a group. It is, however, necessary that there is a composition law for its representations reflecting that a transformation law for fields induces the transformation law for products of fields. For quantum groups (or, more generally, Hopf or even quasiHopf algebras) the composition law is provided by the co-product, comprising the tensor product for groups as a special case.

In the present article, a new attempt is made to overcome the problem of positivity arising with quantum groups. Our construction is explicitly realized on a positive Hilbert space, but the nature of its symmetry is not a priori imposed. Instead, our starting point is the requirement that the charged fields generate the superselection sectors, with the tacit expectation that this determines the symmetry to some extent. We establish a much larger symmetry algebra $\mathscr{M}$ which one may with some reason speculate to contain a quasi-Hopf algebra. The notion of representations for $\mathscr{M}$ is replaced by homomorphisms

$$
\left(\rho_{I J}\right)_{I J}: \mathscr{M} \rightarrow \operatorname{Mat}_{N}(\mathscr{M})
$$

defining $\mathscr{M}$-linear transformations as in (1.13) below, and the composition law

$$
(\rho \times \rho)_{I K, J L}=\rho_{I J}{ }^{\circ} \rho_{K L}
$$

further generalizes the co-product prescription (cf. (1.20)). 
Although the term "plektons" suggests massive excitations in two- or threedimensional physics, our (purely algebraic) results neither depend on a mass gap nor refer to the dimension of space-time. Thus they also pertain to the positive-energy representations of chiral algebras in rational conformal quantum field theory. In that context, the present article addresses the problem of how to pass from vertex-operator-like charged fields [7] to tensor-like fields [8].

The article is organized as follows. In the remainder of this introduction we present our results. The superselection sectors can be generated by partial isometries, and the transformation rules for the latter are displayed in some detail. The actual construction is contained in Sect. 2 (which is therefore quite technical), as well as a number of corollaries on the structure of the algebra. Some special cases, implications, and problems are exposed in Sect. 3. In particular we discuss the question of the existence of distinguished bases in the special case of permutation group statistics, which would confirm that the DR construction is a subalgebra of our field algebra, and formulate it in a basis independent abstract language referring to the relevant braided (in fact symmetric) inclusion of type $I I_{1}$ factors. In the Appendix, we display our positive but incomplete evidence for the validity of this conjecture in the simplest non-abelian example - this feeding our expectation that in a similar manner in the braid group statistics case a field theory of plektons with quasi-Hopf symmetry has a chance to emerge as a subalgebra.

The technical framework of the article is based on the DHR theory of superselection sectors as introduced in [2] (see also the excellent review on concepts and results [9]), which is most powerful for the type of questions under consideration.

We are going to construct a $C^{*}$ field algebra $\mathscr{F}$ extending the algebra of observables $\mathscr{A} \subset \mathscr{F}$ by charged field operators $\psi_{k}$, which act on the observables $A \in \mathscr{A}$ as charge creating intertwiners:

$$
\psi_{k} A=\rho(A) \psi_{k} .
$$

In the special case of permutation group statistics (where all statistical dimensions $d$ are integers), a solution to (1.1) is given by the DR construction in terms of the Cuntz algebra [10], which is generated by isometries $\psi_{k}, k=1, \ldots, d(\rho)$, with the relations

$$
\sum_{k=1}^{d} \psi_{k} \psi_{k}^{*}=1, \quad \psi_{k}^{*} \psi_{l}=\delta_{l k} .
$$

These field operators carry the symmetry of a compact global gauge group $G$, such as to reproduce the superselection structure of the observables $\mathscr{A}=\mathscr{F}^{G}$.

To keep the present construction for braid group statistics comparable, we shall in particular establish the analogous form of orthonormality and completeness relations consistent with non-integer statistical dimensions, as well as the transformation properties of the multiplet field operators $\psi_{k}$ under an appropriate symmetry algebra and their Clebsch-Gordan expansions and commutation relations. The crucial difference with the Cuntz algebra and its $G \subset U(d)$ symmetry is the appearance of a non-abelian coefficient ring $\mathscr{M}$ commuting with the observables but not with charged fields, in which matrix elements of linear transformations, as well as Clebsch-Gordan coefficients and commutation structure constants take values. In fact, for the special case of permutation group statistics we shall present indications, that for an appropriate choice of basis these coefficients 
take scalar values, such that the Cuntz algebra is recovered and the DR construction arises as a subalgebra of the present (more redundant) field algebra.

Let us now present our results more specifically. Assume that $\mathscr{A}$ has only finitely many superselection sectors (i.e. equivalence classes $\left[\pi_{\alpha}\right]$ of irreducible representations which are locally equivalent to the vacuum representation and have finite statistics [2]). Let $\rho$ be a localized endomorphism of $\mathscr{A}$ equivalent to the direct sum of all its sectors, and denote by $E_{\alpha} \in(\rho \mid \rho)=\rho(\mathscr{A})^{\prime}$ the minimal projections in the commutant of $\rho(\mathscr{A})$ corresponding to the subrepresentation of equivalence class $\left[\pi_{\alpha}\right]$. Denote by $\mathscr{M}$ the hyperfinite von Neumann factor of type $I I_{1}$ obtained as the inductive limit of matrix algebras of intertwiners within $\mathscr{A}$

$$
\mathscr{M}:\left(\rho^{n} \mid \rho^{n}\right) \subset\left(\rho^{n+1} \mid \rho^{n+1}\right),
$$

(cf. (2.1) below) equipped with the trace state $\operatorname{tr}=\lim _{n} \phi^{n}$ generated by the unique standard left-inverse $\phi$ of $\rho$. Then $\mathscr{F}$ will be realized as a $C^{*}$ subalgebra of $\mathscr{A} \otimes \mathscr{M}$. In particular, $\mathscr{A} \subset \mathscr{F}$ will be realized by the injection

$$
i_{\mathscr{A}}(A):=\sum_{\alpha} E_{\alpha} \rho(A) \otimes E_{\bar{\alpha}},
$$

and $\mathscr{M} \subset \mathscr{F}$ will be realized by the injection

$$
i_{\mathscr{M}}(m):=\sum_{\alpha} E_{\alpha} \otimes E_{\bar{\alpha}} \rho(m) .
$$

Obviously, $i_{\mathscr{A}}(\mathscr{A})$ and $i_{\mathscr{M}}(\mathscr{M})$ commute.

Let us first describe the internal degrees of freedom of our charged field multiplets abstractly. The indices $k \in \mathscr{K}$ of $\psi_{k} \in \mathscr{F} \subset \mathscr{A} \otimes \mathscr{M}$ will transform like elements of the irreducible sub-bimodules of the two-sided action of $\mathscr{M}$ on itself by left and right multiplication with $\rho(m)$. These bimodules are realized as linear spaces $\mathscr{K}_{\alpha}:=E_{\alpha} \mathscr{K} \subset \mathscr{K}$, labelled by the superselection sectors of $\mathscr{A}$, with the bimodule action by left multiplication with $\rho(m)$ and right multiplication with $m$, where $\mathscr{K}$ is the inductive limit of finite dimensional intertwiner spaces in $\mathscr{A}$,

$$
\mathscr{K}:\left(\rho^{n+1} \mid \rho^{n}\right) \subset\left(\rho^{n+2} \mid \rho^{n+1}\right),
$$

in the topology of $\mathscr{M}$. (cf. (2.1). Observe that $\mathscr{K}^{*} \mathscr{K}=\mathscr{K} \mathscr{K}^{*}=\mathscr{M}$, and $k_{1}^{*} k_{2}$ may be regarded as an $\mathscr{M}$-valued inner product.) The bimodule tensor product has the orthogonal decomposition

$$
\mathscr{K}_{\alpha} \otimes_{\mathscr{M}} \mathscr{K}_{\beta} \cong \sum_{\gamma} N_{\alpha \beta}^{\gamma} \mathscr{K}_{\gamma},
$$

with $N_{\alpha \beta}^{\gamma}=\operatorname{dim}\left(\rho_{\alpha} \rho_{\beta} \mid \rho_{\gamma}\right)$ the multiplicity of the sector $\left[\pi_{\gamma}\right]$ within the product of sectors $\left[\pi_{\alpha}\right]$ and $\left[\pi_{\beta}\right]$, i.e. the tensor structure of the internal degrees of freedom coincides with the superselection structure of the algebra of observables. There is a unitary action of the statistics operator $\varepsilon(\rho, \rho): \mathscr{K}_{\alpha} \otimes_{\mathscr{M}} \mathscr{K}_{\beta} \rightarrow \mathscr{K}_{\beta} \otimes_{\mathcal{M}} \mathscr{K}_{\alpha}$ permuting the order of tensor factors, and intertwining the respective bimodule actions of $\mathscr{M}$.

The internal structure can be made more explicit in terms of bimodule bases $\left\{b_{I}\right\}$ of $\mathscr{K}_{\alpha}$ labelled by $I$ in finite index sets $\mathscr{J}_{\alpha}$, such that

$$
\sum_{I \in \mathscr{J}_{x}} b_{I} b_{I}^{*}=E_{\alpha}, \quad b_{I}^{*} b_{J}=\delta_{I J} p_{I} \in \mathscr{M}
$$


where $p_{I}$ are projections in $\mathscr{M}$. The von Neumann dimension [11] of the bimodule $\mathscr{K}_{\alpha}$ (with respect to either one-sided action of $\mathscr{M}$ ) is given by the basis-independent quantity

$$
\operatorname{dim}_{\mathscr{M}} \mathscr{K}_{\alpha}=\sum_{I \in \mathscr{J}_{\alpha}} \operatorname{tr}\left(p_{I}\right)=d_{\alpha}
$$

the statistical dimension of the superselection sector $\left[\pi_{\alpha}\right]$. The left action of $m \in \mathscr{M}$ on $b_{J} \in \mathscr{K}$ is then given by the right multiplication of $b_{I}$ (summed over $I \in \mathscr{J}=\bigcup_{\alpha} \mathscr{J}_{\alpha}$,
i.e. a basis of $\mathscr{K}$ ) with

$$
\rho_{I J}(m):=b_{I}^{*} \rho(m) b_{J}
$$

The maps $m \mapsto \rho_{I J}(m)$ define an algebra homomorphism $\mathscr{M} \rightarrow \operatorname{Mat}_{N}(\mathscr{M})$, which actually is a direct sum of homomorphisms, one for either sector $\left(N=|\mathscr{J}|=\sum_{\alpha}\left|\mathscr{J}_{\alpha}\right|\right)$.

There are $\mathscr{M}$-valued Clebsch-Gordan coefficients $\mathscr{C}(K \mid J I)_{e}$, with $e$ labelling the "fusion channels" of (1.6), for the composition of these algebra homomorphisms, satisfying

$$
\sum_{I J} \mathscr{C}(K \mid J I)_{e} \cdot \rho_{J J^{\prime}} \circ \rho_{I I^{\prime}}(m)=\sum_{K^{\prime}} \rho_{K K^{\prime}}(m) \cdot \mathscr{C}\left(K^{\prime} \mid J^{\prime} I^{\prime}\right)_{e}
$$

Now let us turn to the field algebra itself. Its existence with the properties listed below is the content of the main Theorem 2.7 and its Corollaries 2.8-2.11.

The charged fields $\psi_{k} \in \mathscr{F}$ fulfill the required charge-creating intertwiner relation (1.1). ${ }^{1}$ Actually, for $k \in \mathscr{K}_{\alpha}, \psi_{k}$ generate the subrepresentation of class $\left[\pi_{\alpha}\right]$ within $\pi_{0}^{\circ} \rho . \psi_{k}$ are linear in $k \in \mathscr{K}$ and transform like

$$
m \psi_{k} n=\psi_{\rho(m) k n}, \quad k \in \mathscr{K} .
$$

In terms of a basis (1.7), the field operators $\psi_{I}=\psi_{b_{I}}$ are partial isometries satisfying the orthonormality and completeness relations in $\mathscr{F}$,

$$
\sum_{I \in \mathscr{J}} \psi_{I} \psi_{I}^{*}=1_{\mathscr{F}}, \quad \psi_{I}^{*} \psi_{J}=\delta_{I J} p_{I} \in i_{\mathscr{M}}(\mathscr{M})
$$

The transformation law may then be rewritten

$$
m \psi_{J}=\sum_{I \in \mathscr{J}} \psi_{I} \rho_{I J}(m)
$$

There is a Wigner-Eckart formula for the reduction of products of tensor operators of the present quantum symmetry according to $(1,6)$ :

$$
\psi_{I} \psi_{J}=\sum_{e, K} T_{e} \cdot \psi_{K} \cdot \mathscr{C}(K \mid J I)_{e}
$$

where $T_{e} \in\left(\rho^{2} \mid \rho\right)$ are observables intertwining the corresponding representations of $\mathscr{A}$. The field algebra $\mathscr{F}$ is spanned by its elements $A \psi_{I} m$. It has a unit $1_{\mathscr{F}}=i_{\mathscr{A}}\left(1_{\mathscr{A}}\right)=i_{\mathscr{M}}\left(1_{\mathscr{M}}\right)$, and it is closed under the $*$-operation defined in $\mathscr{A} \otimes \mathscr{M}$; in particular, the $*$-conjugates of charged field operators carry the conjugate charge, with coefficients in $i_{\mathscr{M}}(\mathscr{M})$ if expanded in terms of a basis. There is a notion of

\footnotetext{
${ }^{1}$ Here and below, in all operator equations within $\mathscr{F}$, observables $A \in \mathscr{A}$ respectively coefficients $m \in \mathscr{M}$ are understood as their images under $i_{\mathscr{A}}$ respectively $i_{\mathscr{M}}$
} 
localization for field operators (different from the one pertaining to the first factor in $\mathscr{A} \otimes \mathscr{M}$ ), characterized by commutativity with the images under $i_{\mathscr{A}}$ of localized observables. Multiplication with elements of $i_{\mathscr{M}}(\mathscr{M})$, and hence the action of the symmetry (1.6), do not affect the localization. Field operators localized at space-like distance satisfy commutation relations with coefficients again in $i_{\mathscr{M}}(\mathscr{M})$.

A Hilbert space representation of the field algebra is given by the GNS construction from a vacuum state. The vacuum state is given by the restriction to $\mathscr{F}$ of the state on $\mathscr{A} \otimes \mathscr{M}$

$$
\omega(A \otimes m):=d \cdot \omega_{0}\left(W_{0}^{*} A W_{0}\right) \cdot \operatorname{tr}(m),
$$

where $\omega_{0}=\left\langle\Omega, \pi_{0}(\cdot) \Omega\right\rangle$ is the vacuum state on $\mathscr{A}, W_{0}$ is an isometry in $(\rho \mid i d)$ such that $W_{0} W_{0}^{*}=E_{0}$ is the projection onto the vacuum subrepresentation of $\rho$, and $d=d(\rho)=\sum_{\alpha} d_{\alpha}=\left(\operatorname{tr} E_{0}\right)^{-1}$. Restricted to the observables, $\omega$ coincides with $\omega_{0}$ :

$$
\omega \circ i_{\mathscr{A}}=\omega_{0} .
$$

On this ground state, the operators $\Gamma_{k}=\psi_{k}^{*} i_{, \mathcal{g}}\left(W_{\alpha}\right)$ with $k \in \mathscr{K}_{\alpha}$ and isometries $W_{\alpha} \in\left(\rho \mid \rho_{\alpha}\right)$, act as creation operators for the representation $\pi_{\alpha}=\pi_{0}^{\circ} \rho_{\alpha}$ of $\mathscr{A}$ :

$$
\omega\left(\Gamma_{k}^{*} \cdot A \Gamma_{k}\right)=\frac{\operatorname{tr}\left(k^{*} k\right)}{d_{\alpha}}\left\langle\Omega, \pi_{\alpha}(A) \Omega\right\rangle .
$$

Consequently, the Hilbert space $\mathscr{H}$ of $\mathscr{F}$ decomposes with respect to the observables like

$$
\mathscr{H}=\bigoplus_{\alpha} \mathscr{H}_{\alpha} \otimes \mathscr{K}_{\bar{\alpha}},
$$

where $\mathscr{H}_{\alpha}$ carry the representations $\pi_{\alpha}$, and $\mathscr{K}_{\bar{\alpha}}$ arise as multiplicity spaces.

We consider the above transformation of fields under $\mathscr{M}$ as a "master symmetry," too redundant for practical purposes. In particular, the elements of $i_{\mathscr{M}}(\mathscr{M})$ play the double role of "coefficients" and "symmetry operators," while a distinction among the two would be more conventional: usually the effect of the transformation with an operator (commutator, adjoint, or more general) is expressible in terms of numerical coefficients. The reduction of the "master symmetry" to a more conventional symmetry may be exemplified by our scenario, how the DR construction is contained in $\mathscr{F}$ in the case of integer statistical dimensions. The idea is to identify a basis $\left\{b_{I}\right\}$ of isometries (i.e. all $p_{I}=1 . \mu$ and hence, by (1.8), $\left|\mathscr{J}_{\alpha}\right|=d_{\alpha}$; such bases exist by an argument of [12]), for which moreover all $\mathscr{C}(K \mid J I)_{e}$ turn into scalars, and to determine the compact symmetry group $G$ as the subgroup of those unitaries in $\mathscr{M}$ for which

$$
\rho_{I J}(u) \doteq g_{I J} \cdot u \quad \text { with } \quad g_{I J} \in \mathbb{C},
$$

(divided by the kernel of $\rho_{I J}$, which is a normal subgroup). Then, (1.13) turns into the usual linear transformation law with representation matrices $g_{I J}$, and (1.10) becomes the usual defining property of Clebsch-Gordan coefficients of a group. In particular, (1.14) rewritten in the form

$$
\sum_{I J K} \psi_{K} \psi_{I}^{*} \psi_{J}^{*} \mathscr{C}(K \mid J I)_{e}=T_{e}^{*} \in i_{\mathscr{A}}(\mathscr{A}),
$$

shows that the $G$-invariant elements of the Cuntz algebra are observables. The 
cohomological problem is, of course, to find a basis with the stated properties (see Sect. 3.2).

More generally, one may hope to identify a Hopf algebra (or quasi-Hopf algebra, as the non-commuting Clebsch-Gordan coefficients may account for deviation from co-associativity by conjugation with operators in $\mathscr{M}$, cf. also [5]) $\mathscr{X}$ within $\mathscr{M}$ such that restricted to $\mathscr{X}$

$$
\rho_{I J} \doteq\left(\tau_{I J} \otimes i d\right) \circ \Delta,
$$

where $\Delta$ is the co-product and $\tau$ is a matrix representation of $\mathscr{X}$. Then (1.13) becomes the commutation relation of the (quasi-) Hopf algebra with field operators in a linear representation of the former.

We want to stress that $\mathscr{M}, \mathscr{K}$, and $\rho$, and consequently the quantum symmetry encoded in (1.9), are given in terms of the superselection structure of $\mathscr{A}$ alone. Mathematically, $\mathscr{M}$ is the "path model" (see e.g. [11]) derived from an incidence matrix which is the sum of the fusion matrices of all sectors of $\mathscr{A}$. The endomorphism $\rho: \mathscr{M} \rightarrow \mathscr{M}$ is a "parallel transport" [13] on $\mathscr{M}$. Since $\mathscr{K}_{\alpha}$ are the equivalence classes of irreducible sub-bimodules of the inclusion $\rho(\mathscr{M}) \subset \mathscr{M}$, the equivalence class of the homomorphism (1.9) may be considered as an invariant for this inclusion in the sense of $[14,13]$. For instance,

$$
\sum_{I \in \mathscr{J}} \operatorname{tr}\left(\rho_{I I}\left(1_{\mathscr{M}}\right)\right)=\sum_{I} \operatorname{tr}\left(p_{I}\right)=\sum_{\alpha} d_{\alpha}=d(\rho)
$$

is the square root of the index $[\mathscr{M}: \rho(\mathscr{M})]$, compare also [15].

In fact, crucial for our construction is also the existence of the statistics operator and of the conjugate representation. These structures define a strict braided monoidal $C^{*}$ category with conjugates, subobjects, and direct sums [16], whose objects are the endomorphisms of $\mathscr{A}$ with non-degenerate statistics, and whose arrows are the intertwiners in $\mathscr{A}$. The inclusion $\rho(\mathscr{M}) \subset \mathscr{M}$ and the trace are completely determined by the sub-category with objects $\rho^{n}$. The braided structure supplies a "metric" $\zeta_{e \bar{e}}$ (Lemma 2.5), which is the only non-standard ingredient for our construction, and which will not exist for general inclusions of factors. $\zeta_{e \bar{e}}$ is related to the antilinear and charge conjugating operation $\dagger$ on intertwiners [9]. Thus, detached from the present context of algebraic quantum field theory (i.e. restricted to the subalgebra $\mathscr{F} \cap \mathscr{M} \otimes \mathscr{M}$ ), our results may be regarded as a statement about special inclusions of type $I I_{1}$ factors and their bimodules, which are naturally associated with braided $C^{*}$ categories. An application of the intertwiner calculus is the fact that the trace $\operatorname{tr}$ on $\mathscr{M}$ provides a topological "ribbon invariant" [17] with "colors" taking values in the irreducible $\rho_{\alpha}$ and their products. In this context, $\zeta_{e \bar{e}}$ is related to a ribbon move that rotates a "coupon" by $180^{\circ}$. Finally, we mention that the abstract intertwiner calculus derived from the DHR theory has its equivalent in the context of conformal field theory in the famous polynomial identities [18] for braiding ( $R$ below) and fusion $(D)$ matrices.

\section{The Construction}

In this section, we shall give the field algebra $\mathscr{F}$ explicitly as a subalgebra of $\mathscr{A} \otimes \mathscr{M}$. The algebraic relations in $\mathscr{F}$ announced in the introduction are derived from identities of the intertwiner calculus for localized endomorphisms, i.e. for the super- 
selection structure of the algebra of observables [2,9]. Let us first recall some (standard) notations.

Representations of the algebra of observables satisfying the selection criterion [2] are given in terms of localized endomorphisms $\rho: \mathscr{A} \rightarrow \mathscr{A}$ with a natural composition law. For any two localized endomorphisms, the linear space of intertwiners from $\rho_{1}$ to $\rho_{2}$ is denoted (opposite to the category notation $\left(\rho_{1}, \rho_{2}\right)$ ) by

$$
\left(\rho_{2} \mid \rho_{1}\right):=\left\{T \in \mathscr{A} \mid T \rho_{1}(A)=\rho_{2}(A) T\right\} .
$$

$(\rho \mid \rho)=\rho(\mathscr{A})^{\prime}$ is the commutant of the endomorphism $\rho$ and reduces to the scalars $\mathbb{C}$ iff $\rho$ is irreducible. In particular, for $\rho_{1}$ irreducible, there is a positive definite inner product on $\left(\rho_{2} \mid \rho_{1}\right)$ given by $T^{*} T^{\prime} \in\left(\rho_{1} \mid \rho_{1}\right)=\mathbb{C}$.

For any pair of localized endomorphisms there are two unitary statistics operators

$$
\left.\varepsilon\left(\rho_{1}, \rho_{2}\right) \text { and } \varepsilon\left(\rho_{2}, \rho_{1}\right)^{*} \in\left(\rho_{2} \rho_{1}\right\rfloor \rho_{1} \rho_{2}\right),
$$

which coincide in the special case of permutation group statistics, but are distinct in the general case of braid group statistics. The latter case is relevant when there is a topological distinction between the "left" and the "right" causal complements of a space-time region of localization. Whenever $\rho_{i}$ are localized at space-like separation and the localization region of $\rho_{1}$ lies to the right of that of $\rho_{2}$, then $\varepsilon\left(\rho_{1}, \rho_{2}\right)=1$.

An endomorphism $\rho$ with finite statistics possesses a conjugate $\bar{\rho}$ and a unique standard left-inverse $\phi: \mathscr{A} \rightarrow \mathscr{A}$, which can be given by the formula

$$
\phi(A)=R^{*} \bar{\rho}(A) R,
$$

with an appropriate isometry $R \in(\bar{\rho} \rho \mid i d)$. The statistical dimension $d$ of $\rho$ is the number $\|\phi(\varepsilon(\rho, \rho))\|^{-1}<\infty$.

The identities valid among generic intertwiners $T$, statistics operators $\varepsilon$, and $R \in(\bar{\rho} \rho \mid i d)$ as a consequence of the basic physical assumptions of algebraic quantum field theory [2] are precisely the axioms of a strict braided monoidal $C^{*}$ category with conjugates, subobjects, and direct sums [16], whose objects are the endomorphisms of $\mathscr{A}$ with finite statistics, and whose arrows are the intertwiners in $\mathscr{A}$. In particular, the semi-group $\Delta$ of localized endomorphisms with finite statistics is closed under composition, and every $\rho \in \Delta$ has a finite decomposition into irreducible $\rho_{\alpha} \in \Delta$. We shall select a collection $\nabla=\left\{\rho_{\alpha}\right\} \subset \Delta$, containing one representative per superselection sector, with $\rho_{0}=i d \in \nabla$.

We shall from now on assume that $\nabla$ is finite. This is mainly for technical reasons, and would amount in the case of permutation group statistics [1] to the restriction to theories with finite symmetry groups. In the context of conformal quantum field theories with $\mathscr{A}=$ the algebra of chiral observables, it is the restriction to "rational" theories [18].

We pick $\rho \in \Delta$ equivalent to the direct sum of $\rho_{\alpha} \in \nabla$. Then $\rho(\mathscr{A})^{\prime}$ is a finite abelian $C^{*}$ algebra with minimal projections $E_{\alpha}=W_{\alpha} W_{\alpha}^{*}$ onto the irreducible components, $W_{\alpha} \in\left(\rho \mid \rho_{\alpha}\right)$ isometries. We denote $\varepsilon=\varepsilon(\rho, \rho), \stackrel{\alpha}{\mathscr{T}}=\left(\rho^{2} \mid \rho\right), \mathscr{R}=\left(\rho^{2} \mid i d\right)$. Since $\rho$ is self-conjugate, we choose $\bar{\rho}=\rho$ and $R \in \mathscr{R}$ such that (2.3) gives the standard left-inverse $\phi$ of $\rho$. Then the following useful formulae hold:

$$
E_{\bar{\alpha}} R=\rho\left(E_{\alpha}\right) R,
$$




$$
\begin{gathered}
\phi\left(E_{\alpha}\right)=d_{\alpha} / d, \quad \phi\left(R R^{*}\right)=d^{-2}, \\
\phi^{n}(T A)=d^{m-n} \cdot \phi^{m}(A T) \text { for } T \in\left(\rho^{n} \mid \rho^{m}\right),
\end{gathered}
$$
where $d_{\alpha}=d\left(\rho_{\alpha}\right)$ and $d=d(\rho)=\sum_{\alpha} d_{\alpha}$ denote the statistical dimensions. We pick a
basis of $\mathscr{T}$

$$
T_{e} \in E_{\alpha} \rho\left(E_{\beta}\right) \cdot \mathscr{T} \cdot E_{\gamma}=W_{\alpha} \rho_{\alpha}\left(W_{\beta}\right) \cdot\left(\rho_{\alpha} \rho_{\beta} \mid \rho_{\gamma}\right) \cdot W_{\gamma}^{*} \subset \mathscr{T}
$$

where $e$ stands for the "superselection channel of type $(\alpha, \beta, \gamma)$ " of $\rho_{\gamma}$ contained in $\rho_{\alpha} \rho_{\beta}$, or in other words, of the transition from the sector $[\alpha]$ to the sector $[\gamma]$ effectuated by the charge $[\beta]$. The number of basis elements of a given type is $N_{\alpha \beta}^{\gamma}=\operatorname{dim}\left(\rho_{\alpha} \rho_{\beta} \mid \rho_{\gamma}\right)$. The basis can be chosen such that

$$
\sum_{e} T_{e} T_{e}^{*}=1 \in\left(\rho^{2} \mid \rho^{2}\right), \quad T_{e}^{*} T_{f}=\delta_{e f} E_{\gamma} .
$$

We fix the intertwiners for the special channels involving the vacuum sector ${ }^{2}$ :

$$
\begin{array}{ll}
T_{s(\alpha)}=W_{0} E_{\alpha} & \text { for } \quad e=s(\alpha) \text { of type }(0, \alpha, \alpha), \\
T_{t(\alpha)}=\rho\left(W_{0}\right) E_{\alpha} & \text { for } \quad e=t(\alpha) \text { of type }(\alpha, 0, \alpha), \\
T_{r(\alpha)}=\left(d_{\alpha} / d\right)^{-1 / 2} \cdot E_{\bar{\alpha}} R W_{0}^{*} & \text { for } \quad e=r(\alpha) \text { of type }(\bar{\alpha}, \alpha, 0) .
\end{array}
$$

Now, before we construct the field algebra, we quote a series of technical, more or less standard lemmas, the proofs of which may be omitted or only sketched.

Lemma 2.1. $\rho(\mathscr{A})^{\prime}=(\rho \mid \rho)=\bigoplus_{\alpha} \mathbb{C} E_{\alpha}$, while for $n>1$, the subalgebras $\rho^{n}(\mathscr{A})^{\prime}=$ $\left(\rho^{n} \mid \rho^{n}\right)$ are spanned by $T_{e_{1}} \ldots T_{e_{n-1}}^{\alpha} T_{e_{n-1}^{\prime}}^{*} \ldots T_{e_{1}^{\prime}}^{*}$. By virtue of $(2.6)$ and $(2.5)$, the map $\phi^{n}$ is a trace on $\left(\rho^{n} \mid \rho^{n}\right)$, converging to a trace on $\mathscr{M}$ given by (1.2). In particular, $\mathscr{M}$ is the path algebra [11] induced from the incidence matrix $N_{\alpha}^{\gamma}=\sum_{\beta} N_{\alpha \beta}^{\gamma}$.

Lemma 2.2. $(\rho \mid$ id $)=\mathbb{C} W_{0}$, while for $n>1$, the linear spaces $\left(\rho^{n+1} \mid \rho^{n}\right)$ are spanned by $T_{e_{1}} \cdots T_{e_{n}} T_{e_{n-1}^{\prime}}^{*} \cdots T_{e_{1}^{\prime}}^{*}$, which are orthogonal with respect to the scalar product $\left(T_{1}, T_{2}\right)=\operatorname{tr}\left(T_{1}^{*} T_{2}\right)$. Defining $\mathscr{K}$ as in (1.5) (in the topology induced from $\mathscr{M}$ by the identification $\left.k \mapsto k W_{0}^{*} \in \mathscr{M}\right)$ and $\mathscr{K}_{\alpha}=E_{\alpha} \mathscr{K} \subset \mathscr{K}, \mathscr{K}_{\alpha}$ are equivalent to the inequivalent irreducible sub-bimodules of the two-sided action of $\mathscr{M}$ on itself induced by the endomorphism $\rho: \mathscr{M} \rightarrow \mathscr{M}$. Every bimodule basis (1.7) of $\mathscr{K}_{\alpha}$ satisfies the dimension formula (1.8).

Proof of the last two statements. Consider the two-sided action of $\mathscr{M}$ on itself induced by $\rho$, i.e. the action by left and right multiplication with $\rho(m)$. This action is naturally equivalent with the action on $\rho(\mathscr{M}) \mathscr{R}$ by left multiplication with $\rho^{2}(m)$ and right multiplication with $m$. Since the commutant of the latter two-sided action is left multiplication with $\left(\rho^{2} \mid \rho^{2}\right)$, the irreducible sub-bimodules are given by $E_{\alpha} \cdot \mathscr{T}^{*} \rho(\mathscr{M}) \mathscr{R}=\mathscr{K}_{\alpha}$. By (2.6) we have $\operatorname{tr} p_{I}=d \cdot \operatorname{tr} b_{I} b_{I}^{*}$, hence (1.8) by (2.5).

Lemma 2.3. Every element $m \in \mathscr{M}$ has a unique decomposition

$$
m=\sum_{e} T_{e}^{*} \rho\left(k_{e}\right)
$$

with $k_{e} \in \mathscr{K}_{\beta}$ for e of type $(\alpha, \beta, \gamma)$.

${ }^{2}$ Requiring the normalization of phases $W_{0}^{*} R=d^{-1 / 2} W_{0}$ 
Proof. Since $R^{*} \rho(R)$ is an invertible element of $(\rho \mid \rho) \subset \mathscr{M}$, and $1 \in \mathscr{T}^{*}$, we get

$$
\begin{aligned}
\mathscr{M} \subset \mathscr{M} \mathscr{R}^{*} \rho(\mathscr{R}) & =\mathscr{R}^{*} \rho^{2}(\mathscr{M}) \rho(\mathscr{R}) \subset \mathscr{R}^{*} \rho\left(\mathscr{T}^{*}\right) \rho^{2}(\mathscr{M}) \rho(\mathscr{R}) \subset \mathscr{T}^{*} \rho\left(\mathscr{T}^{*} \rho(\mathscr{M}) \mathscr{R}\right) \\
& \subset \mathscr{T}^{*} \rho(\mathscr{K}) \subset \mathscr{M},
\end{aligned}
$$

proving the existence of an expansion (2.10). Uniqueness is proven by applying $\phi\left(T_{f} \cdots\right)$ to (2.10) and noting that $\phi\left(T_{f} T_{e}^{*}\right) \in(\rho \mid \rho) \cdot E_{\beta}$ must be a multiple of $E_{\beta}$, which in turn with (2.5) and (2.6) is seen to be proportional to $\delta_{e f}$.

Lemma 2.4. The following finite expansions with scalar biunitary coefficient matrices $D, R$ hold.

$$
\begin{aligned}
\rho\left(T_{f}\right) & =\sum_{e, e_{1}, e_{2}} D_{f ; e}^{e_{1} e_{2}} \cdot T_{e_{1}} T_{e_{2}} T_{e}^{*}, \\
\rho\left(\varepsilon^{ \pm 1}\right) & =\sum_{e_{1}, e_{2}, e_{1}^{\prime}, e_{2}^{\prime}} R_{e_{2}^{\prime} e_{1}^{\prime}}^{e_{1} \circ e_{2}}( \pm) \cdot T_{e_{1}} T_{e_{2}} T_{e_{1}^{\prime}}^{*} T_{e_{2}^{\prime}}^{*}
\end{aligned}
$$

$D$ and $R$ vanish unless $e_{1}, e_{2}, e, f, e_{2}^{\prime}, e_{1}^{\prime}$ are of respective type $\left(\alpha, \rho_{1}, \beta\right),\left(\beta, \rho_{2}, \gamma\right)$, $\left(\alpha, \rho_{3}, \gamma\right),\left(\rho_{1}, \rho_{2}, \rho_{3}\right),\left(\alpha, \rho_{2}, \beta^{\prime}\right),\left(\beta^{\prime}, \rho_{1}, \gamma\right)$.

In the language of [13], (2.12) provides the parallel transport in the path algebra $\mathscr{M}$. Bi-unitary is the validity of two (pairs of equivalent) identities, which we display here only for $D$ :

$$
\begin{gathered}
\sum_{e_{1}, e_{2}}\left[D_{f ; e}^{e_{1} e_{2}}\right]^{*} D_{f^{\prime} ; e^{\prime}}^{e_{1} \circ e_{2}}=\delta_{e e^{\prime}} \delta_{f f^{\prime}} \Leftrightarrow \sum_{e, f}\left[D_{f ; e}^{e_{1} \circ e_{2}}\right]^{*} D_{f ; e}^{e_{1}^{\prime} \circ e_{2}^{\prime}}=\delta_{e_{1} e_{1}^{\prime}} \delta_{e_{2} e_{2}^{\prime}}, \\
\sum_{e_{2}, e} d_{\gamma}\left[D_{f ; e}^{e_{1} \circ e_{2}}\right]^{*} D_{f_{1}^{\prime} ; e}^{e_{1}^{\prime} \circ e_{2}}=\frac{d_{\beta} d_{\rho_{3}}}{d_{\rho_{1}}} \delta_{e_{1} e_{1}^{\prime}} \delta_{f f^{\prime}} \Leftrightarrow \sum_{e_{1}, f} d_{\rho_{1}}\left[D_{f ; e}^{e_{1} \circ e_{2}}\right]^{*} D_{f ; e^{\prime}}^{e_{1} e_{2}^{\prime}}=\frac{d_{\beta} d_{\rho_{3}}}{d_{\gamma}} \delta_{e_{2} e_{2}^{\prime}} \delta_{e e^{\prime}}
\end{gathered}
$$

For the case of a group symmetry, we shall recognize the coefficients $D$ as the $6 j$ symbols (see (3.15)).

Proof. Define $D$ by $D_{f ; e}^{e_{1} e_{2}} \cdot E_{\gamma}=T_{e_{2}}^{*} T_{e_{1}}^{*} \rho\left(T_{f}\right) T_{e}$, and $R$ analogously. Equation (2.8) then implies (2.12), (2.13) as well as unitarity of $D$ (the first line of (2.14)) and $R$. Biunitarity of $D$ (the second line of (2.14)) and $R$ is more tedious and involves (2.5) and (2.6).

Lemma 2.5. There are invertible coefficient matrices $\zeta_{e \bar{e}}$ implementing the symmetries

$$
\begin{gathered}
\zeta_{e_{1} \bar{e}_{1}} \zeta_{e_{2} \bar{e}_{2}} \cdot D_{\bar{f} ; \bar{e}}^{\bar{e}_{1} \circ \bar{e}_{2}}=\left[D_{f ; e}^{e_{1} \circ e_{2}}\right]^{*} \cdot \zeta_{f \bar{f}} \zeta_{e \bar{e}}, \\
\zeta_{e_{1} \bar{e}_{1}} \zeta_{e_{2} \bar{e}_{2}} \cdot R_{\bar{e}_{2}^{\prime} \circ \bar{e}_{1}^{\prime}}^{\bar{e}_{1} \circ \bar{e}_{2}}( \pm)=\left[R_{e_{2}^{\prime} \circ e_{1}^{\prime}}^{e_{1} \circ e_{2}}(\mp)\right]^{*} \cdot \zeta_{e_{2}^{\prime} \bar{e}_{2}^{\prime}} \zeta_{e_{1}^{\prime} \bar{e}_{1}^{\prime}},
\end{gathered}
$$

with summations over repeated indices understood. $\zeta_{e \bar{e}}$ are given by the scalar operators in $($ id $\mid$ id $)=\mathbb{C}$,

$$
\zeta_{e \bar{e}}=\left(\frac{\omega_{\alpha} \omega_{\beta}}{\omega_{\gamma}}\right)^{1 / 2}\left(\frac{d_{\alpha} d_{\beta} d_{\gamma}}{d^{3}}\right)^{-1 / 2} \cdot R^{*} R^{*} \rho\left(\varepsilon^{*}\right) T_{e} \rho\left(T_{\bar{e}}\right) R
$$


which vanish unless for e of type $(\alpha, \beta, \gamma), \bar{e}$ is of the conjugate type $(\bar{\alpha}, \bar{\beta}, \bar{\gamma}) .^{3}$ They satisfy

$$
\zeta_{e \bar{e}} \zeta_{e^{\prime} \bar{e}}^{*}=\delta_{e e^{\prime}} \frac{d_{\gamma}}{d_{\alpha} d_{\beta}}
$$

and take the special values $\zeta_{s(\alpha) s(\bar{\alpha})}=\zeta_{t(\alpha) t(\bar{\alpha})}=1$ and $\zeta_{r(\alpha) r(\bar{\alpha})}=d_{\alpha}^{-1}$.

The proof can be found in [19]. ${ }^{4}$ From the previous lemmas we obtain

Proposition 2.6. Let $\zeta:=\sum_{e \bar{e}} \zeta_{e \bar{e}} \cdot \frac{d_{\alpha} d_{\beta}}{d_{\gamma}} \cdot T_{e}^{*} \otimes T_{\bar{e}}^{*} \in \mathscr{T}^{*} \otimes \mathscr{T}^{*}$. Then

$$
\begin{gathered}
\zeta \cdot(\rho \otimes \rho)(\zeta)=\zeta \cdot \zeta, \\
\zeta \cdot \zeta \cdot(\rho(\varepsilon) \otimes 1)=\zeta \cdot \zeta \cdot(1 \otimes \rho(\varepsilon)), \\
\zeta^{*}=d \cdot \zeta \cdot(\rho(R) \otimes \rho(R)) .
\end{gathered}
$$

Proof. (2.19) is just the TPC relation (2.15) and the unitarity of $D$. Equation (2.20) is the TPC relation (2.16) and unitarity of $R$. Equation (2.21) is (2.15) and bi-unitarity of $D$. To see the latter, introduce the invertible operator $X=R^{*} \rho(R) \in \rho(\mathscr{A})^{\prime}$ and expand, following the spirit of (2.11),

$$
T_{e}=\sum_{f, g} R^{*} \rho\left(T_{g}\right) T_{f} \cdot T_{f}^{*} \cdot \rho\left(T_{g}^{*} \rho\left(T_{e}\right) R\right) \cdot X^{-1} .
$$

Retracing the effect of the projections $E$ going along with $T$ by (2.7), we find that for $e$ of type $(\alpha, \beta, \gamma)$, only $f$ of type $(\gamma, \bar{\beta}, \alpha)$ and $g$ of type $(\bar{\gamma}, \alpha, \bar{\beta})$ contribute to the sum. Next we expand by (2.12)

$$
T_{g}^{*} \rho\left(T_{e}\right) R=D_{e ; r(\gamma)}^{g \circ r(\beta)} \cdot T_{r(\beta)} T_{r(\gamma)}^{*} R=D_{e ; r(\gamma)}^{g \circ r(\beta)} \cdot\left(d_{\gamma} / d_{\beta}\right)^{1 / 2} E_{\bar{\beta}} R
$$

Here only the special channels $r(\beta)$ and $r(\gamma)$, cf. (2.9), contribute, and (2.3-5) are used; similarly,

$$
R^{*} \rho\left(T_{g}\right) T_{f}=D_{g ; f}^{r(\bar{\gamma}) \circ s(\alpha)} \cdot R^{*} T_{r(\bar{\gamma})} T_{s(\alpha)}=D_{g ; f}^{r(\bar{\gamma}) o s(\alpha)} \cdot\left(d_{\gamma} / d\right)^{1 / 2} E_{\alpha}
$$

Putting everything together, the projections can be absorbed in $T_{f}$, and doing the same manipulations for both tensor factors of $\zeta^{*}$, we get

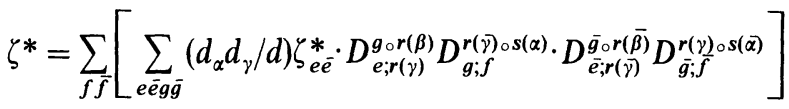

$$
\begin{aligned}
& \cdot T_{f}^{*} \rho(R) X^{-1} \otimes T_{f}^{*} \rho(R) X^{-1} .
\end{aligned}
$$

The numerical coefficient $[\cdots]$ here can be evaluated by using $(2.15)$ and the

${ }^{3}$ This justifies the interpretation of (2.15) and (2.16) as a "TPC" symmetry.

4 To avoid confusion we stress that $T_{e} \in\left(\rho^{2} \mid \rho\right)$ in (2.7) differ from what was called $T_{e} \in\left(\rho_{\alpha} \rho_{\beta} \mid \rho_{\gamma}\right)$ in [19] by the intertwiners $W$. This facilitates the presentation at the price of some obvious changes in the formulae. The present $\zeta_{e \bar{e}} \operatorname{differ}$ from $\zeta(e)$ in [19] by the convenient factors $\omega^{1 / 2}$, which do not affect the symmetries 
bi-unitarity (2.14) of $D$ twice. It then simplifies to

$$
d^{-1} \cdot \frac{d_{\beta} d_{\gamma}}{d_{\alpha}} \zeta_{f \bar{f}}
$$

The operators $X^{-1}=\left[R^{*} \rho(R)\right]^{-1} \in \rho(\mathscr{A})^{\prime}$ in (2.22) hit on the projection $E_{\gamma}$ of $T_{f}$ in the first, and on $E_{\bar{\gamma}}$ in the second tensor factor. Since $\rho(\mathscr{A})^{\prime}$ is abelian, they just contribute scalar coefficients $1 / x, 1 / \bar{x}$, given by $X E_{\gamma}=x E_{\gamma}, X E_{\bar{\gamma}}=\bar{x} E_{\bar{\gamma}}$. We compute

$$
x \bar{x} E_{\bar{\gamma}}=x R^{*} \rho(R) E_{\bar{\gamma}}=x R^{*} \rho\left(E_{\gamma}\right) \rho(R)=R^{*} \rho\left(X E_{\gamma} R\right),
$$

and apply $R^{*} \ldots R$, using $R^{*} R^{*}=R^{*} \rho^{2}\left(R^{*}\right)$ :

$$
x \bar{x} R^{*} E_{\bar{\gamma}} R=\phi\left(\rho\left(R^{*}\right) X E_{\gamma} R\right)=\phi\left(E_{\gamma} X \rho\left(R^{*}\right) R\right)=\phi\left(E_{\gamma} \phi\left(R R^{*}\right)\right)=d^{-2} \phi\left(E_{\gamma}\right) .
$$

Then, $R^{*} E_{\bar{\gamma}} R=R^{*} \rho\left(E_{\gamma}\right) R=\phi\left(E_{\gamma}\right) \neq 0$ implies $1 / x \bar{x}=d^{2}$. This proves (2.21).

Now we can turn to the construction of the field algebra.

Theorem 2.7. For $A \in \mathscr{A}, k \in \mathscr{K}$ define the operator $\varphi(A, k) \in \mathscr{A} \otimes \mathscr{M}$ as the (basis independent) sum

$$
\varphi(A, k):=\sum_{e, \bar{e}} \frac{d_{\alpha}}{d_{\gamma}} \zeta_{e \bar{e}} \cdot T_{e}^{*} \rho(A) \otimes T_{\bar{e}}^{*} \rho(k) .
$$

The linear span of operators $\varphi(A, k)$ is closed under multiplication and *-conjugation:

$$
\begin{aligned}
\varphi\left(A_{1}, k_{1}\right) \varphi\left(A_{2}, k_{2}\right) & =\sum_{f, \bar{f}} \zeta_{f \vec{f}} \varphi\left(A_{f}, k_{\bar{f}}\right), \\
\varphi(A, k)^{*} & =\varphi\left(A^{+}, k^{+}\right),
\end{aligned}
$$

where $A_{f}=T_{f}^{*} \rho\left(A_{1}\right) A_{2} \in \mathscr{A}, k_{\bar{f}}=T_{\bar{f}}^{*} \rho\left(k_{1}\right) k_{2} \in \mathscr{K}$, and $A^{+}=d^{1 / 2} \rho\left(A^{*}\right) R \in \mathscr{A}, k^{+}=$ $d^{1 / 2} \rho\left(k^{*}\right) R \in \mathscr{K}$. The $C^{*}$ closure of the algebra spanned by $\varphi(A, k)$ is the field algebra $\mathscr{F}$. The unit operator in $\mathscr{F}$ is $1_{\mathscr{F}}=\sum_{\alpha} E_{\alpha} \otimes E_{\bar{\alpha}}$.

$\varphi(A, k)$ is said to carry charge $\beta$ if $k \in \mathscr{K}_{\beta}$. Observe that then only $e$ of type $(\cdot, \bar{\beta}, \cdot)$ contribute to the sum (2.23), and hence

$$
\varphi\left(A, E_{\beta} k\right)=\varphi\left(E_{\bar{\beta}} A, k\right) .
$$

Obviously, for $\varphi \in \mathscr{F}$ carrying charge $\beta$, its conjugate $\varphi^{*}$ carries the conjugate charge $\bar{\beta}$, and for $\varphi_{i}$ carrying charges $\beta_{i}$, the expansion (2.24) of $\varphi_{1} \varphi_{2}$ contains only contributions from operators with charges $\beta$ such that $\rho_{\beta}$ is contained in $\rho_{\beta_{1}} \rho_{\beta_{2}}$.

Note the structure of a crossed product underlying the $\mathscr{A}$ part of (2.24)!

Proof. The proof is immediate from Proposition 2.6, if we note that (2.23) may be rewritten as

$$
\varphi(A, k)=\zeta \cdot\left(\rho(A) \otimes \rho\left(\left(\sum_{\beta} d_{\beta}^{-1} E_{\beta}\right) k\right) .\right.
$$

Proposition 2.8. The special elements of $\mathscr{F}$ (carrying zero charge),

$$
\begin{aligned}
& i_{\mathscr{A}}(A):=\varphi\left(W_{0} A, W_{0}\right) \\
& i_{\mathscr{M}}(m):=\varphi\left(W_{0}, W_{0} m\right)
\end{aligned}
$$


coincide with (1.3) respectively (1.4) and satisfy

$$
\begin{aligned}
B \cdot \varphi(A, k) \cdot C & =\varphi(\rho(B) A C, k), & & B, C \in \mathscr{A}, \\
m \cdot \varphi(A, k) \cdot n & =\varphi(A, \rho(m) k n), & & m, n \in \mathscr{M} .
\end{aligned}
$$

The special charged elements

$$
\psi_{k}:=\varphi(R, y \cdot k)
$$

with $y:=\sum_{\beta} \sqrt{d d_{\beta}} E_{\beta} \in \rho(\mathscr{A})^{\prime}$, fulfill the intertwining relation (1.1). Therefore they commute with observables at space-like distance (where $\rho$ acts trivially). They satisfy the transformation law (1.11), as well as the orthogonality and completeness relations

$$
\begin{gathered}
\psi_{k_{1}}^{*} \psi_{k_{2}}=k_{1}^{*} k_{2} \in i_{\mathscr{M}}(\mathscr{M}), \\
\sum_{i} \psi_{k_{i}} \psi_{k_{i}^{\prime}}^{*}=m \in i_{\mathscr{M}}(\mathscr{M}) \quad \text { whenever } \quad \sum_{i} k_{i} k_{i}^{\prime *}=\rho(m) .
\end{gathered}
$$

For a basis (1.7), these take the form (1.12) and (1.13).

For $k \in \mathscr{K}_{\alpha}$, i.e. $\psi_{k}$ carrying charge $\alpha$, one has $E_{\alpha} \psi_{k}=\psi_{k}$, and $W_{\alpha}^{*} \psi_{k}$ is an intertwiner for $\rho_{\alpha}$. In particular, $\psi_{0}:=\psi_{W_{0}}=W_{0} \in i_{\mathscr{A}}(\mathscr{A})$, and all $\psi_{k}$ carrying charge zero are of the form $i_{\mathscr{A}}\left(W_{0}\right) \cdot i_{\mathscr{M}}(m)$.

Proof. The first statement is evident, since due to $W_{0}$ only $e=t(\alpha)$ contribute to (2.23). Equation (2.29) follows from (2.24) using (2.9), (1.1) and (1.11) follow from (2.29). To verify (2.31), we compute $\psi_{k_{1}}^{*} \psi_{k_{2}}$ with (2.25) and (2.24), and observe that due to $\rho\left(R^{+}\right) R \in \mathscr{R}$ only $T_{f}=T_{r(\alpha)}$ contribute. This simplifies the result to

$$
\psi_{k_{1}}^{*} \psi_{k_{2}}=\sum_{\alpha} d^{2} / d_{\alpha}^{2} \cdot \varphi\left(W_{0} \cdot R^{*} R^{*} \rho(R) E_{\bar{\alpha}} R, W_{0} \cdot k_{1}^{*} y^{*} R^{*} \rho(R) E_{\alpha} y k_{2}\right) .
$$

As in the final part of the proof of Proposition 2.6, we have $y^{*} R^{*} \rho(R) E_{\alpha} y=d_{\alpha} d x E_{\alpha}$ and $R^{*} R^{*} \rho(R) E_{\bar{\alpha}} R=\bar{x} \phi\left(E_{\alpha}\right)=\left(d_{\alpha} / d\right) \bar{x}$ with $x \bar{x}=d^{-2}$, which gives (2.31). To verify (2.32) from (2.24) and (2.25), we observe that to the $\mathscr{M}$ part $T_{f}^{*} \rho\left(y \rho(m) y^{*}\right) R=$ $T_{\bar{f}}^{*} \rho\left(y y^{*}\right) R \cdot m$ only $\bar{f}=r(\bar{\alpha})$ contribute, and use (2.4), (2.5), and (2.9). The rest follows immediately from the definitions.

Corollary 2.9. For a bimodule basis (1.7) and $\psi_{I} \equiv \psi_{b_{I}}$, the operators

$$
A \cdot \psi_{I} \cdot m=\varphi\left(\rho(A) R, y b_{I} m\right), \quad A \in \mathscr{A}, I \in \mathscr{J}, m \in \mathscr{M}
$$

span $\mathscr{F}$. The following expansion holds (with appropriate $k_{e}$ )

$$
\psi_{k_{1}} \psi_{k_{2}}=\sum_{e} T_{e} \psi_{k_{e}}
$$

where for $\psi_{k_{i}}$ carrying charge $\beta_{i}$ only e of type $\left(\beta_{1}, \beta_{2}, \beta\right)$ and $\psi_{k_{e}}$ carrying charge $\beta$ contribute. In particular, for bases $b_{I} \in \mathscr{K}_{\beta_{1}}, b_{J} \in \mathscr{K}_{\beta_{2}}, b_{K} \in \mathscr{K}_{\beta}$ as in (1.7) the expansion (1.14) holds with coefficients in $\mathscr{M}$,

$$
\mathscr{C}(K \mid J I)_{e}=b_{K}^{*} \tilde{T}_{e}^{*} \rho\left(b_{I}\right) b_{J} \quad \text { with } \quad \tilde{T}_{e}=\left(\omega_{\beta} / \omega_{\beta_{1}} \omega_{\beta_{2}}\right)^{1 / 2} \cdot \varepsilon^{*} T_{e},
$$

which we call Clebsch-Gordan, since they decompose product representations of the

${ }^{5}$ Recall footnote 1 
symmetry in the sense of (1.10). Finally, with $k^{+}$as in (2.25),

$$
\psi_{k}^{*}=d^{1 / 2} R^{*} \cdot \psi_{k^{+}}
$$

Proof. The first statement is obvious since $\rho(\mathscr{A}) R=\mathscr{A}[3]$ and $b_{I}$ are a right module basis. Equation (2.34) follows from

$$
T_{e}^{*} \psi_{k_{1}} \psi_{k_{2}}=\sum_{f \bar{f}} \zeta_{f \bar{f}} \cdot \varphi\left(\rho\left(T_{e}^{*}\right) T_{f}^{*} \rho(R) R, T_{\bar{f}}^{*} \rho\left(y k_{1}\right) y k_{2}\right) .
$$

The $\mathscr{K}$ entry vanishes unless $\bar{f}$ is of type $\left(\beta_{2}, \beta_{1}, \beta\right)$, and is an element of $\mathscr{K}_{\beta}$. Then $f$ is of type $\left(\bar{\beta}_{2}, \bar{\beta}_{1}, \bar{\beta}\right)$, and the $\mathscr{A}$ entry vanishes unless $e$ is of type $\left(\beta_{1}, \beta_{2}, \beta\right)$. The $\mathscr{A}$ entry, being an element of $E_{\bar{\beta}} \mathscr{R}$, is a multiple of $E_{\bar{\beta}} R$, and by virtue of $\varphi\left(E_{\bar{\beta}} R, k\right)=\varphi\left(R, E_{\beta} k\right)$ all contributions sum up to a term $\varphi\left(R, y k_{e}\right)=\psi_{k_{e}}$ with $k_{e} \in \mathscr{K}_{\beta}$. Working out the details yields $k_{e}=\tilde{T}_{e}^{*} \rho\left(k_{1}\right) k_{2}$, hence $(2.35)$ for $k_{1}=b_{I}$ and $k_{2}=b_{J}$ basis elements. It is an elementary check, that these coefficients have the property (1.10). Equation (2.36) follows easily from the definitions, (2.25), and $(y k)^{+}=y \cdot k^{+}$.

Corollary 2.10. The ground state (1.15) takes the form

$$
\omega\left(A \cdot \psi_{k}\right)=\omega_{0}\left(A W_{0}\right) \cdot \operatorname{tr}\left(W_{0}^{*} k\right) .
$$

The n-point function for generic charged operators $\varphi_{i}=\Gamma_{k_{i}} A_{i} \in \mathscr{F}$, where $\Gamma_{k}=\psi_{k}^{*} W_{\alpha}, k \in \mathscr{K}_{\alpha}, A \in i_{\mathscr{A}}(\mathscr{A})$, reads

$$
\begin{aligned}
\omega\left(\varphi_{n} \cdots \varphi_{1}\right)= & \sum_{\eta=e_{1} \cdots e_{n}} \omega_{0}\left(t_{e_{n}}^{*} \rho_{\gamma_{n-1}}\left(A_{n}\right) t_{e_{n-1}}^{*} \cdots t_{e_{2}}^{*} \rho_{\gamma_{1}}\left(A_{2}\right) A_{1}\right) \\
& \cdot \operatorname{tr}\left(W_{0}^{*} \tilde{T}_{e_{n}}^{*} \rho\left(\cdots \tilde{T}_{e_{3}}^{*} \rho\left(\tilde{T}_{e_{2}}^{*} \rho\left(k_{1}\right) k_{2}\right) k_{3} \cdots\right) k_{n}\right)^{*} .
\end{aligned}
$$

$e_{i}$ are of type $\left(\gamma_{i-1}, \alpha_{i}, \gamma_{i}\right), \gamma_{0}=\gamma_{n}=0$, standing for the subsequent interpolation of the fields carrying charge $\alpha$ between sectors $\gamma$, and $t_{e_{i}}=\rho\left(W_{\alpha_{i}}^{*}\right) W_{\gamma_{1}-1}^{*} T_{e_{i}} W_{\gamma_{i}} \in$ $\left(\rho_{\gamma_{i}-1} \rho_{\alpha_{i}} \mid \rho_{\gamma_{i}}\right), t_{e_{1}}=1 .^{6}$ The vacuum-to-vacuum amplitude $\omega_{0}(\cdots)$ depends only on the local degrees of freedom and the involved sectors, but neither on the kind nor the values of the symmetry degrees of freedom attached to fields: in the case of an ordinary group symmetry, they coincide with the reduced amplitudes of $n$-point functions of tensor operators (in the Wigner-Eckart sense), e.g., those given by the DR construction. In the general case they coincide with the full $n$-point function of the operators $\left(e_{i}, A_{i}\right)$ of the reduced field bundle [3], which is a construction completely deprived of inner degrees of freedom. The dependence on the symmetry degrees of freedom $k$ is entirely in the amplitude $\operatorname{tr}(\cdots)$, which (for $k_{i}=b_{I_{i}}$ basis vectors) may be expanded as polynomials in the Clebsch-Gordan coefficients (using $\tilde{T}_{e}^{*} \rho\left(b_{I}\right) b_{J}=\sum_{K} b_{K} \mathscr{C}(K \mid J I)_{e}$ ) and their images under $\rho_{L M}$ (using $\rho(m)=$ $\left.\sum_{L M} b_{L} \rho_{L M}(m) b_{M}^{*}\right)$. In particular, if a basis can be identified for which all $\mathscr{C}(K \mid J I)_{e}$ are scalars (see Sect. 3.2), these polynomials coincide with the usual expressions for the invariant tensor amplitudes in terms of Clebsch-Gordan coefficients.

Proof. Equation (2.37) is easily computed from the definitions (2.30), (2.23), using (2.29) and (2.4-9). Using (1.1) and (2.34) repeatedly (with $k_{e}$ as specified in the proof

${ }^{6}$ Recall footnote 4; the present $t_{e}$ are the $T_{e}$ of $[3,19]$ 
of Corrollary 2.9) yields

$$
\varphi_{n} \cdots \varphi_{1}=\sum_{e_{2} \cdots e_{n}} \psi_{k_{\eta}}^{*} \cdot T_{e_{n}}^{*} \cdots T_{e_{2}}^{*} \rho^{n-1}\left(W_{\alpha_{n}} A_{n}\right) \cdots \rho\left(W_{\alpha_{2}} A_{2}\right) W_{\alpha_{1}} A_{1}
$$

with $k_{\eta}=\tilde{T}_{e_{n}}^{*} \rho\left(\tilde{T}_{e_{n-1}}^{*}\right) \cdots \rho^{n-2}\left(\tilde{T}_{e_{2}}^{*}\right) \rho^{n-1}\left(k_{1}\right) \cdots \rho\left(k_{n-1}\right) k_{n}$. Then (2.38) follows from (2.37).

Let us now turn to the notion of localization. A field operator $\varphi(A, k)$ is said to be localized in a (double-cone) region $\mathcal{O}$ if it commutes with all observables localized in the casual complement $\mathcal{O}^{\prime}$. As in [3] it is easily seen that then $A=U^{*} C$ with $C \in \mathscr{A}(\mathcal{O}), U$ unitary, and $\hat{\rho}(\cdot)=U \rho(\cdot) U^{*}$ localized in $\mathcal{O}$. In particular, $\psi_{k}$ are localized in the localization region of $\rho$, and $i_{\mathscr{A}}(U) \psi_{k}$, which are intertwiners for $\hat{\rho}$, are localized in $\mathcal{O}$. Actually, $\mathscr{F}(\mathcal{O})=\bigoplus_{I} i_{\mathscr{A}}(\mathscr{A}(\mathcal{O}) U) \psi_{I} i_{\mathscr{M}}(\mathscr{M})$.

Proposition 2.11. Let $\varphi_{I}=U_{1} \psi_{I}$ be localized in $\mathcal{O}_{1}$, and $\varphi_{J}^{\prime}=U_{2} \psi_{J}$ in $\mathcal{O}_{2}$. Then the following commutation relations hold:

$$
\begin{aligned}
\varphi_{I} \varphi_{J}^{\prime} & =\varepsilon\left(\rho_{1}, \rho_{2}\right)^{*} \sum_{J^{\prime} I^{\prime}} \varphi_{J^{\prime}}^{\prime} \varphi_{I^{\prime}} \cdot \mathscr{R}_{J I}^{I^{\prime} J^{\prime}}(+) \\
& =\varepsilon\left(\rho_{2}, \rho_{1}\right) \sum_{J^{\prime} I^{\prime}} \varphi_{J^{\prime}}^{\prime} \varphi_{I^{\prime}} \cdot \mathscr{R}_{J I}^{I^{\prime} J^{\prime}}(-),
\end{aligned}
$$

where $\rho_{i}(\cdot)=U_{i} \rho(\cdot) U_{i}^{*}$. In particular, when $\mathcal{O}_{i}$ are at space-like distance and $\mathcal{O}_{1}$ is to the right of $\mathcal{O}_{2}$, then $\varepsilon\left(\rho_{1}, \rho_{2}\right)=1$ and the first formula gives the commutation relation with coefficients $\mathscr{R}(+) \in \mathscr{M}$, while for $\mathcal{O}_{1}$ to the left of $\mathcal{O}_{2}, \varepsilon\left(\rho_{2}, \rho_{1}\right)=1$ and the second formula gives the commutation relation with coefficients $\mathscr{R}(-) \in \mathscr{M}$. The coefficients are given by

$$
\begin{aligned}
\mathscr{R}_{J I}^{I^{\prime} J^{\prime}}( \pm) & =b_{I^{\prime}}^{*} \rho\left(b_{J^{\prime}}^{*}\right) \cdot \varepsilon^{ \pm 1} \cdot \rho\left(b_{I}\right) b_{J} \\
& \equiv \sum_{e e^{\prime} K} \mathscr{C}(K \mid J I)_{e}^{*} R_{s\left(\alpha_{2}\right)_{\circ} e^{\prime}}^{s\left(\alpha_{1}\right)_{e}}( \pm) \mathscr{C}\left(K \mid I^{\prime} J^{\prime}\right)_{e^{\prime}}
\end{aligned}
$$

Proof. It is sufficient to prove (2.39) for $U_{i}=1, \rho_{i}=\rho$, since $U_{1} \rho\left(U_{2}\right) \varepsilon(\rho, \rho)=$ $\varepsilon\left(\rho_{2}, \rho_{1}\right) U_{2} \rho\left(U_{1}\right)$. Thus we compute $\varepsilon \cdot \psi_{I} \psi_{J}$. With (2.3.), (2.24), (2.29) we get

$$
\sum_{f \bar{f}} \zeta_{f \bar{f}} \cdot \varphi\left(\rho(\varepsilon) T_{f}^{*} \rho(R) R, T_{\bar{f}}^{*} \rho\left(y b_{I}\right) y b_{J}\right)
$$

We use $\rho^{2}(\varepsilon) \rho(R) R=\rho\left(\varepsilon^{*}\right) R R=\varepsilon \rho(R) R$ and $\zeta \cdot(\varepsilon \otimes 1)=\zeta \cdot(1 \otimes \varepsilon)$, which is $(2.20)$ after right multiplication with $W_{0} \otimes W_{0}$. This gives

$$
\sum_{g \bar{g}} \zeta_{g \bar{g}} \cdot \varphi\left(T_{g}^{*} \rho(R) R, T_{\bar{g}}^{*} \varepsilon \rho\left(y b_{I}\right) y b_{J}\right)
$$

$y \rho(y)$ commutes with $\varepsilon$. Finally expanding $\varepsilon \rho\left(b_{I}\right) b_{J}=\sum_{I^{\prime} J^{\prime}} \rho\left(b_{J^{\prime}}\right) b_{I^{\prime}} b_{I^{\prime}}^{*} \rho\left(b_{J^{\prime}}^{*}\right) \varepsilon \rho\left(b_{I}\right) b_{J}$, we obtain the first equation (2.39) with $\mathscr{R}(+)$ given by $(2.40)$. The calculation is identical for the second equation (2.39).

We have thus established the existence of a $C^{*}$ field algebra with very analogous properties as shared by the DR construction, except that its transformation rules, operator product expansions, and commutation relations come with noncommuting instead of scalar coefficients. 


\section{Special Bases and Special Cases}

In this section, we want to discuss the program of finding subalgebras on which a more conventional symmetry is realized, for some special instances. The most important question is whether for permutation group statistics the usual gauge group symmetry, which is known to exist independently [1], is in fact realized within our "master symmetry." Although we cannot prove this in general, we discuss the consistency of this scenario by a number of structural cross-checks in Sect. 3.2. The answer is positive for an abelian gauge group, and is extended to abelian braid group statistics (anyons). Here, a new and apparently typical feature of quantum symmetry in low-dimensional systems shows up in its most transparent version: the symmetry may become implemented by inner automorphisms of the field algebra. This is discussed in Sect. 3.1. Finally we show in Sect. 3.3 that the "exchange algebra" (with its structure constant matrices on "path spaces" instead of tensor spaces [3]) is also contained in the present construction.

3.1. The Abelian Case (Anyons). Let us consider for simplicity a theory with $\mathbb{Z}_{\mathrm{N}}$ superselection structure

$$
\nabla=\left\{\alpha^{n} \mid n=0, \ldots, N-1\right\}, \quad \rho_{\alpha}^{N} \cong i d .
$$

As is well known, the statistics phases are $\omega\left(\rho_{\alpha}^{n}\right)=\omega^{n^{2}}$, implying $\omega^{N}= \pm 1$. The sign is + if $N$ is odd. It is also + in the special case of permutation group statistics. Let us first assume the sign to be + . This is equivalent $[2,19]$ to the existence of a representative $\rho_{\alpha} \in[\alpha]$ for which $\rho_{\alpha}^{N}=i d$, which in turn amounts to the existence of a choice of basis $T_{e} \equiv T_{i j}=\rho\left(W_{j}\right) W_{i} W_{i+j}^{*}$ for $e$ of type $\left(\alpha^{i}, \alpha^{j}, \alpha^{i+j}\right)$ (with addition always $\bmod N$ ), for which all non-vanishing matrix elements $D_{f ; e}^{e_{1} e_{2}}=1$. Thus

$$
\rho\left(T_{i j}\right)=\sum_{k} T_{k i} T_{(k+i) j} T_{(i+j) k}^{*}
$$

Now put

$$
\begin{aligned}
b_{i} & =\sum_{j} T_{i j} \in \mathscr{K}_{i}, \\
u & =\sum_{k l} e^{-2 \pi i k / N} T_{k l} T_{k l}^{*} \equiv \sum_{k} e^{-2 \pi i k / N} E_{k} \in \mathscr{M} .
\end{aligned}
$$

We immediately verify

$$
\begin{gathered}
b_{i} b_{i}^{*}=E_{i}, \quad b_{i}^{*} b_{j}=\delta_{i j}, \\
u^{*} u=u u^{*}=1, \quad u^{N}=1, \\
\rho_{j k}(u)=\delta_{j k} e^{2 \pi i k / N} \cdot u, \\
\mathscr{C}(k \mid j i)=\delta_{k(i+j)}, \\
\mathscr{R}_{j i}^{i^{\prime} j^{\prime}}( \pm)=\omega^{ \pm i j} \cdot \delta_{i i^{\prime}} \delta_{j j^{\prime}},
\end{gathered}
$$

where for the last two statements we use $\varepsilon\left(\rho_{\alpha}, \rho_{\alpha}\right)=\omega$, hence $\varepsilon\left(\rho_{\alpha}^{i}, \rho_{\alpha}^{j}\right)=\omega^{i j}$. This confirms the desired transformation properties of $\psi_{i}$ under the group $\mathbb{Z}_{\mathrm{N}}$ implemented by $u \in \mathscr{M}$, as well as the anyonic commutation relations at space-like 
distance (with notation as in (2.39))

$$
\varphi_{i} \varphi_{j}^{\prime}=\omega^{ \pm i j} \varphi_{j}^{\prime} \varphi_{i}
$$

Observe that the subalgebra generated by the observables and $\psi_{i}$ is spanned by $A \cdot \psi_{i}$, and hence the symmetry operators $u^{n}$ act as outer automorphisms on this subalgebra. This is the usual situation with global gauge transformations implemented by global charges which are not in the algebra of localized fields.

In contrast, in the case with an obstruction [19]: $N$ even, $\omega^{N}=-1$ (which is excluded for permutation group statistics), one obtains non-scalar $\mathscr{C}(k \mid j i)$ with values in the group ring of $\mathbb{Z}_{\mathrm{N}} \in \mathscr{M}$, while the transformation properties given by (3.7) remain unchanged. At least for $N=2$ and 4, the coefficients in the sum (3.3) may be adjusted such that all Clebsch-Gordan coefficients are scalar multiples of group elements. Thus the fields transform in the usual way, but the multiplication law for charged fields lets the unitaries $u^{n}$ implementing the symmetry be in the algebra generated by $\mathscr{A}$ and $\psi_{i}$. The symmetry is implemented by inner automorphisms, and, since the vacuum vector obtained from the ground state (1.15) is separating, it cannot be invariant under $u$ : instead there is an $N$-fold degenerate vacuum. One may envisage another ground state on this subalgebra (realized in $\left.\mathscr{A} \otimes\left(\rho^{2} \mid \rho^{2}\right)\right)$ by replacing $\operatorname{tr}(m)$ in $(1.15)$ by $d^{-2} W_{0}^{*} W_{0}^{*} m W_{0} W_{0}$, which gives rise to an invariant but not separating vacuum vector.

In fact, the issue is independent of our present construction: just postulating $\psi$ to satisfy the intertwining relation (1.1), the DHR theory predicts the commutation relations $(3.10)$ for $i$ or $j$ or $i-j=0$. Then $\left(\varphi_{1}\right)^{N}$ carries zero charge but anticommutes with $\varphi_{1}^{\prime}$. This already excludes scalar $\mathscr{C}$.

It is thus impossible to maintain a sharp distinction between "local" and "global" aspects: $u$ are generated from localized operators, but have the global property of non-trivially transforming charged operators at any localization. This seems to be a characteristic feature of the quantum symmetry. It is most clearly exhibited in our example of abelian braid group statistics with an obstruction (such models are explicitly constructed in [20]; in fact, there the group ring valued coefficients were already encountered, but - in the context of chiral algebras on the circle - had to be considered as elements of $\mathscr{A}$ ), while it is also implicit in all attempts to implement non-abelian Hopf or quasi-Hopf algebra symmetries on operator algebras (e.g. [5]).

3.2. The Case of Permutation Group Statistics. In the case of permutation group statistics, the DR construction [1] yields a field algebra, which extends the observables $\mathscr{A}$ by intertwining isometries $\psi_{I}$ with the properties (1.1), (1.12), (1.14) with $p_{I}=1$ and $\mathscr{C}(K \mid J I)_{e}$ scalars, as well as a group $G$ represented by unitaries $\mathscr{U}(g)$ on the Hilbert space, under which $\psi_{I}$ transform as finite multiplets such that $\mathscr{C}(K \mid J I)_{e}$ are the Clebsch-Gordan coefficients of the corresponding unitary representation matrices $\mathscr{D}_{I J}(g) \in \mathbb{C}$. This algebra is constructed abstractly and does not involve $\mathscr{M}$ like ours.

- We conjecture that the DR construction is a subalgebra of our construction.

This conjecture has been confirmed above for abelian symmetry groups. In other words, we expect isometries $b_{I} \in \mathscr{K}_{\alpha}$ and unitaries $u(g) \in \mathscr{U}(\mathscr{M})$ to solve the following 
equations in $\mathscr{M}$ :

$$
\begin{gathered}
\sum_{I \in \mathscr{J}_{\alpha}} b_{I} b_{I}^{*}=E_{\alpha}, \quad b_{I}^{*} b_{j}=\delta_{I J}, \\
b_{K}^{*} \tilde{T}_{e}^{*} \rho\left(b_{I}\right) b_{J} \equiv \mathscr{C}(K \mid J I)_{e} \in \mathbb{C}, \\
u\left(g_{1}\right) u\left(g_{2}\right)=u\left(g_{1} g_{2}\right), \\
b_{I}^{*} \rho(u(g)) b_{J} \equiv \rho_{I J}(u(g))=\mathscr{D}_{I J}(g) \cdot u(g) .
\end{gathered}
$$

(Actually, (3.13) needs to hold only up to a cocycle $z$ commuting with $\psi_{I}$, i.e. $\rho_{I J}(z)=\delta_{I J} \cdot z$.) If these conditions are fulfilled, then the $C^{*}$ algebra generated by $\mathscr{A}$ and $\psi_{I}$ will never involve elements of $\mathscr{M}$ other than scalars. We emphasize that these conditions are sufficient to identify $\psi_{I}$ algebraically with those given by [1] (possibly up to a Klein transformation) and the corresponding subalgebra of $\mathscr{F}$ with $\mathscr{F}^{\mathrm{DR}}$, and to turn (1.14) and (2.38) into the familiar Wigner-Eckart formulae.

In fact, the existence of such bases is an intricate cohomological problem, where the major part of the mathematical depth of the DR result has been shifted into in the present approach. Essentially, (3.12) provides the concrete representation spaces $\operatorname{span}\left\{b_{I} \mid I \in \mathscr{J}_{\alpha}\right\}$ together with their intertwiners $T_{e}$, i.e. the prerequisites for the Tannaka-Krein theorem. We shall present part of an explicit construction for the smallest non-abelian group $\mathrm{S}_{3}$ in Appendix.

The following properties are automatically shared by solutions to (3.11-14). We take the tight self-consistency and consistency with the DR result which they reflect as support, though not proof, for our conjecture.

- The $C^{*}$ subalgebra of $\mathscr{F}$ generated by $\mathscr{A}$ and $\psi_{I}$ is spanned by $\mathscr{A} \psi_{I}$. Namely, the condition $\mathscr{C}(K \mid J I)_{e} \in \mathbb{C}$ implies (putting $J=0 \in \mathscr{J}_{0}$ ) that $b_{0}=W_{0}$ up to a phase and $b_{I}$ are isometries: $p_{I}=1$, and (putting $K=0$ ) that $\operatorname{span}\left\{b_{I}^{+} \mid I \in \mathscr{J}_{\alpha}\right\}=$ $\operatorname{span}\left\{b_{J} \mid J \in \mathscr{J}_{\bar{\alpha}}\right\}$, hence $\psi_{I}^{*} \in \operatorname{span}\left\{R^{*} \psi_{J}\right\}$ by (2.36).

- Given a basis of isometries, the unitaries of $\mathscr{M}$ satisfying (1.19) form a group with unitary representation matrices $\mathscr{D}_{I J}(u)$. The unitaries with $\mathscr{D}_{I J}(u)=\delta_{I J}$ form a normal subgroup, and $\mathscr{D}_{I J}$ is a faithful representation of the quotient group $G$. $\mathscr{D}$ decomposes as a direct sum of subrepresentations $\mathscr{D}^{\alpha}$ for $I, J \in \mathscr{J}_{\alpha}$. Equations (3.14) and (1.10) imply that the scalars $\mathscr{C}(K \mid J I)_{e}$ are the group theoretical Clebsch-Gordan coefficients of $G$. In particular, $\mathscr{D}^{0}$ for $[0]=[i d]$ is the trivial representation, and $\mathscr{D}^{\bar{\alpha}}$ and $\mathscr{D}^{\alpha}$ are conjugate representations. Finally, the corresponding field operators transform in the conventional way:

$$
u \psi_{J} u^{*}=\sum_{I} \psi_{I} \mathscr{D}_{I J}(u)
$$

- For bases of isometries, the size $\left|\mathscr{J}_{\alpha}\right|$ of the multiplet of charge $\alpha$, i.e. the dimension of the corresponding representation of the symmetry group, is given by the statistical dimension $d_{\alpha} \in \mathbb{N}$. This is evident from (1.8).

- $\operatorname{tr} \circ u$ is the $\delta$-function on $G$ : summing (3.14) over $I=J \in \mathscr{J}_{\alpha}$ and applying tr, with (2.6), (3.11), and (2.5) yields $d_{\alpha} \cdot \operatorname{tr}(u(g))=\sum_{I \in \mathscr{J}_{x}} \mathscr{D}_{I I}(g) \cdot \operatorname{tr}(u(g))$. Now, $\sum_{I \in \mathscr{I}_{x}} \mathscr{D}_{I I}(g)$, the character of the representation $\mathscr{D}^{\alpha}$, differs from its dimension $d_{\alpha}$ unless $g$ is the group unit, hence $\operatorname{tr}(u(g))=\delta_{g e}$.

- As in the DR construction, the coefficients $D$ of the parallel transport are nothing 
but the group theoretical recoupling constants (6j-symbols). Namely, comparing the two possibilities to expand $\rho^{2}\left(b_{I}\right) \rho\left(b_{J}\right) b_{K}$ in terms of $b_{M}$ by using (3.12) in the form $\rho\left(b_{I}\right) b_{J}=\sum_{K e} \tilde{T}_{e} b_{K} \mathscr{C}(K \mid J I)_{e}$ twice yields

$$
\delta_{L L^{\prime}} \cdot D_{h ; g}^{e_{o} f}=\sum_{I J K M N} \mathscr{C}(L \mid K M)_{f} \mathscr{C}(M \mid J I)_{e} \mathscr{C}\left(L^{\prime} \mid N I\right)_{g}^{*} \mathscr{C}(N \mid K J)_{h}^{*} .
$$

- The commutation matrix $\mathscr{R}_{J I}^{I^{\prime} J^{\prime}}$ differs from the "canonical" graded permutation matrix $\pm \delta_{I I} \delta_{J J^{\prime}}$ (with entries -1 if both $I$ and $J$ belong to a fermionic sector, +1 else) at most by a similarity transformation, corresponding to some Klein transformation for the field operators $\psi$. Namely, $\mathscr{R}$ is a unitary $G$-invariant matrix with scalar entries (by (2.40)), and defines a matrix representation of $S_{n}$ on $\left(\bigoplus_{\alpha} \mathbb{C}^{d_{\alpha}}\right)^{\otimes n}$ in the commutant of the action of $G$. The character of this representation can be calculated by inserting the first formula (2.40) for $\mathscr{R}$, applying $\operatorname{tr}$ (since everything is scalar, this does not change the result), and using (2.6) and completeness of $b_{I}$. This yields $d^{n}$ times the trace tr of the corresponding operator representative in terms of $\varepsilon\left(\right.$ e.g. $\left.\sum_{I, J \in \mathscr{f}_{\alpha}} \mathscr{R}_{J I}^{J I}=d^{2} \operatorname{tr}\left(\varepsilon E_{\alpha}\right)=\omega_{\alpha} d_{\alpha}\right)$, which in turn is known to coincide with the character of the canonical representation of $S_{n}$.

We want to include a proof that a basis of isometries in $\mathscr{K}_{\alpha}$ always exists, and hence (1.1) can be solved by the generators of a Cuntz subalgebra. To this end, we study the inclusion $\mathscr{N}=\rho(\mathscr{M}) \subset \mathscr{M}$ and the corresponding "basic construction" [14] $\mathscr{M} \subset \mathscr{M}_{1}=\langle\mathscr{M}, e\rangle$, where $e$ is the projection on $L^{2}(\mathscr{N}) \subset L^{2}(\mathscr{M})$. The argument is just an adaptation of an argument given in [12]: for $g_{i} \in \mathscr{M}_{1}$ a partition of unity into projections of $\operatorname{trace} \operatorname{tr}\left(g_{i}\right)=[\mathscr{M}: \mathcal{N}]^{-1}=d^{-2}=\operatorname{tr}(e)$ there are partial isometries $v_{i} \in \mathscr{M}_{1}$ such that $v_{i}^{*} v_{i}=e, v_{i} v_{i}^{*}=g_{i}$, and $m_{i} \in \mathscr{M}$ such that $v_{i}=m_{i} e$. Now, since all the factors are isomorphic to the unique hyperfinite type $I I_{1}$ factor, it is convenient to identify $\mathscr{M}_{1}$ with $\mathscr{M}$ such that $e \in \mathscr{M}_{1}$ is represented as $R R^{*} \in \mathscr{M}$. This identification maps the subfactors $\mathscr{M} \subset \mathscr{M}_{1}$ into $\rho(\mathscr{M}) \subset \mathscr{M}$ and $\mathscr{N} \subset \mathscr{M}$ into $\rho^{2}(\mathscr{M}) \subset \rho(\mathscr{M})$. The Pimsner-Popa basis above is thus given by $v_{i}=\rho\left(m_{i}\right) R R^{*}$. Now it suffices to choose the partition of unity $g_{i}$ to be finer than the partition of unity by $E_{\beta} \rho\left(E_{\alpha}\right)$, the traces of which are $d_{\alpha} d_{\beta} / d^{2}=$ integer multiples of $\operatorname{tr}\left(g_{i}\right)$. Then there are precisely $d_{\alpha}$ projections $g_{I}$ among the $g_{i}$ which are majorized by $E_{0} \rho\left(E_{\alpha}\right)$. The corresponding $m_{I}$ are in $E_{\alpha} \mathscr{M} E_{0}$. Setting $b_{I}=W_{0}^{*} \rho\left(m_{I}\right) R=d^{-1 / 2} m_{I} W_{0} \in \mathscr{K}_{\alpha}$, the properties of $m_{I}$ given in [12] precisely translate into (3.11).

The above also gives rise to a more abstract formulation for the condition (3.12). One may view the subfactor $\rho(\mathscr{M}) \subset \mathscr{M}$ as a special inclusion which is equiped with Jones projections $e_{i}=\rho^{i-1}(e)\left(e=R R^{*}\right)$ and with unitary representatives of the braid group (in this case: permutation group) $\varepsilon_{i}=\rho^{i-1}(\varepsilon)$ satisfying a system of structure identities. Moreover, $\rho(\mathscr{M})^{\prime} \cap \mathscr{M}$ is an abelian $C^{*}$ algebra spanned by its central projections $E_{\alpha}$, there is a notion of label conjugation such that $E_{\alpha} e=\rho\left(E_{\bar{\alpha}}\right) e$, and a distinguished self-conjugate label 0 . In this setting, (3.12) can be equivalently formulated as the condition on $m_{I}=E_{\alpha} m_{I} E_{0}$,

$$
\rho\left(m_{I}\right) \varepsilon \rho\left(m_{J}\right) \stackrel{!}{=} \sum_{K} t_{J I}^{K} \cdot \rho\left(m_{K}\right) \quad \text { with } \quad t_{J I}^{K} \stackrel{!}{\in} \rho^{2}(\mathscr{M})^{\prime} \cap \mathscr{M} .
$$


It seems intriguing that (3.16) has a formal solution if $m_{I}$ were allowed to take values in the homogeneous Cuntz algebra $C_{0} \subset \mathscr{F}^{\text {DR }}$ of which $\mathscr{M}$ is the subalgebra of $G$-invariants [1], corresponding to $b_{I}=\psi_{I}^{\mathrm{DR}}$ formally solving (3.11) and (3.12); but of course this does not help us to construct $\psi_{I}:=\psi_{b_{I}}$ since $\psi_{I}^{\mathrm{DR}}$ are not in $\mathscr{K}$.

3.3. The Exchange Algebra. There is an immediate choice of a bimodule basis of $\mathscr{K}_{\alpha}$ satisfying (1.7), namely the intertwiners $T_{e} \in \mathscr{K}_{\alpha}$ with $e$ of type $(\alpha, \cdot, \cdot)$, or $\widetilde{T}_{e} \in \mathscr{K}_{\alpha}$ with $e$ of type $(\cdot, \alpha, \cdot)$ (see $(2.35))$, which are partial isometries. The corresponding $\psi_{e}$ or $\widetilde{\psi}_{e}$ generate a subalgebra $\mathscr{F}_{\text {red }}$ of $\mathscr{F}$, which is known as the "reduced field bundle" [3] or "exchange algebra" [7]. The operators $F_{e}$ given in [3] essentially coincide with $A \cdot \widetilde{\psi}_{e}$. The range projections $p_{e}$ are given by sector projections $E_{\gamma}$ and thus account for the interpolation properties of exchange field operators.

Actually, $\mathscr{F}_{\text {red }}=\mathscr{F}_{1}$ is the first member of a series of increasing subalgebras $\mathscr{F}_{n} \subset \mathscr{A} \otimes\left(\rho^{n+1} \mid \rho^{n+1}\right)$ with inductive limit $\mathscr{F}$. The entire analysis for $\mathscr{F}$ holds for either $\mathscr{F}_{n}$ except that the appropriate $\mathscr{K}_{n, \alpha}$ are finite dimensional reducible bimodules for the finite dimensional matrix rings $\mathscr{M}_{n}=\left(\rho^{n} \mid \rho^{n}\right)$ with non-trivial center, and there is no chance to find bases with $p_{I}=1$ for non-abelian statistics. In particular, $\mathscr{F}_{\text {red }}$ is completely deprived of the symmetry, since the left and right action of $(\rho \mid \rho) \subset \mathscr{M}$ is trivial; e.g., in the case of an ordinary group symmetry, the $n$-point functions of $\mathscr{F}_{\text {red }}$ are the reduced amplitudes of $n$-point functions of tensor operators, with all Clebsch-Gordan coefficients stripped off in the sense of the Wigner-Eckart theorem.

This example shows how the role of elements of $\mathscr{M}$ can be shifted from the desired one: carriers of the gauge symmetry, to another extremum: mere measurers of the sector (Casimir operators). In order to avoid the occurrence of non-numerical coefficients, the reduced field bundle has sacrificed the irreducibility of the charged multiplets. But in fact, for a decent interpretation as a symmetry, precisely the latter should be an essential aspect.

\section{Conclusion and Discussion}

We presented the construction of a $C^{*}$ field algebra of charged operators, which are intertwiners for the superselection sectors of the subalgebra of observables. The transformation properties of the field operators with respect to an appropriate quantum symmetry satisfy the "duality" requirements between symmetry and statistics [21]. On the other hand, the symmetry algebra has some unusual aspects, which partly are only of formal nature (e.g., we do not deal with matrix representations but rather with homomorphisms into the matrix ring over the algebra), but partly also reflect an intrinsically novel feature of symmetries for low-dimensional quantum field theories. This feature is that operators which implement the global symmetry are algebraically (i.e. not as limits of integrals over densities) generated by localized charged fields. This surprising observation has a bearing at least on issues like the Noether theorem in quantum field theory and on the structure of the ground state, as we discussed for the most explicit example of certain anyonic field theories. These abelian examples show also, that the need of operator-valued structure constants for interpolating fields is a property of superselection sectors with braid group statistics, which generically cannot be avoided by a better choice of fields. It is an intrinsic signal of what is built into most model constructions by 
means of flux lines, contour integrals, or Jordan-Wigner transformations. More fundamentally, it urges us to remember that we should not expect the classical fields of a model to match the physical excitations (charged particle states): the actual local(ization) properties of quantum operators generating the latter may turn out unrelated to how they are described in terms of microscopic degrees of freedom.

A definite advantage of our construction is that it automatically yields a representation of the field algebra on a Hilbert space (the GNS representation induced from the ground state $\omega$ ) thus avoiding all problems with indefinite matrics. The price to pay is a huge redundance of the field algebra reflected in the multiplet structure of infinite complex dimension, to be reduced to finite multiplets in terms of appropriate bases and appropriate subalgebras of our "master symmetry" algebra $\mathscr{M}$. What "appropriate" means here, is not known a priori, but the study of this question can be restricted to the superselection structure and statistics of $\mathscr{A}$ alone, completely detached from the rest of the dynamics. This is the virtue of the separation of the quantum symmetry in terms od Eqs. (1.6-10) from the field algebra relations (1.1) and (1.11-14).

A central problem is the question, how "close to scalar" the Clebsch-Gordan coefficients can be chosen. For non-isometric bases, which are necessary in the case of non-integer statistical dimensions, the condition (3.16) on the Pimsner-Popa basis is equivalent to the property that $\widetilde{T}_{e}^{*} \rho\left(b_{I}\right) b_{J}$ and (provided $\left.m_{0}=E_{0}\right) b_{I}^{+}$are linear combinations of $b_{K}$, i.e. that the span of $\mathscr{A} \cdot \psi_{I}$ closes as a $C^{*}$ subalgebra. On the other hand, resuming the speculation in the introduction about a quasiHopf symmetry, we rather expect $\mathscr{C}(K \mid J I)_{e}$ to factorize into scalar coefficients $c\left(K \mid J^{\prime} I^{\prime}\right)_{e}$ carrying the dependence on $K$ and $e$, and elements $\left(\tau_{I I^{\prime}} \otimes \tau_{J J^{\prime}} \otimes i d\right)(\phi) \in \mathscr{X}$, where the "associator" $\phi \in \mathscr{X} \otimes \mathscr{X} \otimes \mathscr{X}$ controls the deviation from co-associativity [5]. This type of quantum symmetry would signal itself if (3.16) can be solved only with additional coefficients in $\rho^{2}(\mathscr{M})$ multiplying from the right.

Indeed, the obstructed abelian situations with $\omega^{N}=-1$ discussed in Sect. 3.1 correspond to quasi-Hopf algebras, which as algebras and as co-algebras coincide with the Hopf algebras of $\mathbb{Z}_{\mathrm{N}}$ but are equiped with a non-trivial "permutator" $R$ and associator $\phi$. E.g., for $\mathbb{Z}_{2}=\{e, g\}$, putting $p=\frac{1}{2}(e+g)$ and $q=\frac{1}{2}(e-g)$, $R=p \otimes p+q \otimes p+q \otimes p \pm i q \otimes q$ and $\phi=e \otimes e \otimes e-2 q \otimes q \otimes q$ solve the quasiHopf axioms [4], and yield the correct $G$-valued Clebsch-Gordan coefficient $\mathscr{C}(0 \mid 11)=\left(\tau_{1} \otimes \tau_{1} \otimes i d\right)(\phi)=g$. This shows that the quasi-Hopf structure, as it goes beyond the algebra and co-algebra structures, encodes the multiplicative properties of intertwiners generating the representations.

We admit that we have not solved (except for the abelian case) the cohomological problem to reproduce in the special case of permutation group statistics from our construction the DR result of an ordinary (finite) symmetry group. But we see the virtue of our analysis in that it provides a quite natural, for intrinsic, framework to study the issue of quantum symmetry associated with general statistics, without relying on a present from the Heavens (the Cuntz algebra) available for permutation group statistics only.

The present construction is an improved version of an attempt initiated in [21], the major flaw of which was the lack of the $*$-operation. The crucial technique to get the $*$-operation is to contract conjugate charges (in the sense of Theorem 2.7) with a "metric" implementing a rudimental TPC symmetry (Lemma 2.5). It was 
applied before in [19] for an analogous construction with $\mathscr{A} \otimes \mathscr{A}$ instead of $\mathscr{A} \otimes \mathscr{M}$.

Formally, our construction may be viewed as a (diagonal) chiral tensoring of a dynamical theory (the observable physics of interest) with a corresponding "topological field theory" [22]. The degrees of freedom of the latter are purely kinematical data, namely the finite-dimensional spaces of intertwiners between products of the (representatives of) superselection sectors of the observables. This is more fashionably depicted as a functor from surfaces with boundaries labelled by the sectors into vector spaces. In the present context, these degrees of freedom acquire the role of the gauge symmetry for the opposite (dynamical) tensor factor.

\section{Appendix}

We present elements of an explicit solution to (3.11-14) for a specific example, the non-abelian group $\mathrm{S}_{3}=\mathrm{D}_{3}$. It is the group generated by its elements $g_{2}$ and $g_{3}$ with the relations $g_{2}^{2}=g_{3}^{3}=\left(g_{2} g_{3}\right)^{2}=e$. It has three representations of symmetric, anti-symmetric, and mixed symmetry type. Hence, we consider a quantum field theory with three superselection sectors $[0],[\sigma],[\tau]$ of statistical dimensions $1,2,1$ respectively, and with the non-trivial fusion rules

$$
[\tau][\tau]=[0], \quad[\tau][\sigma]=[\sigma], \quad[\sigma][\sigma]=[0]+[\sigma]+[\tau],
$$

consistent with permutation group statistics only if all sectors are bosonic, $\omega_{\alpha}=1$.

We have to solve (3.11-14) within $\mathscr{K}$ and $\mathscr{M}$ with the Clebsch-Gordan coefficients $\mathscr{C}$ and the recoupling constants $D$ determining the parallel transport $\rho$ given by those of $\mathrm{S}_{3}$.

For bases $\psi_{0}, \psi_{ \pm}, \psi_{3}$ of the three representations of $\mathrm{S}_{3}$

$$
\begin{aligned}
\mathscr{D}_{00}\left(g_{2}\right) & =\mathscr{D}_{00}\left(g_{3}\right)=1, \\
\mathscr{D}_{ \pm \mp}\left(g_{2}\right) & =1, \quad \mathscr{D}_{ \pm \pm}\left(g_{3}\right)=e^{ \pm 2 \pi i / 3}, \\
\mathscr{D}_{33}\left(g_{2}\right) & =-1, \quad \mathscr{D}_{33}\left(g_{3}\right)=1,
\end{aligned}
$$

we have the Clebsch-Gordan coefficients

$$
\begin{array}{lll}
\mathscr{C}(J \mid I 0)=\mathscr{C}(J \mid 0 I)=\delta_{I J}, & \mathscr{C}(0 \mid 33)=1, & \mathscr{C}( \pm \mid \pm 3)=\mathscr{C}( \pm \mid 3 \pm)= \pm 1, \\
\mathscr{C}(0 \mid \pm \mp)=2^{-1 / 2}, & \mathscr{C}(3 \mid \mp \pm)= \pm 2^{-1 / 2}, & \mathscr{C}(\mp \mid \pm \pm)=1
\end{array}
$$

(omitting the self-explanatory index $e$ for the relevant channel). These by (3.15) determine the recoupling constants $D$, and hence the action (2.12) of $\rho$ on the basis intertwiners:

$$
\begin{aligned}
& E_{0} \rho(|\alpha(\beta) \gamma\rangle)=|0(\alpha) \alpha(\beta) \gamma\rangle \cdot\langle\gamma(\gamma) 0|, \\
& E_{\alpha} \rho(|\beta(0) \beta\rangle)=\sum_{\gamma}|\alpha(\beta) \gamma(0) \gamma\rangle \cdot\langle\gamma(\beta) \alpha|, \\
& E_{\alpha} \rho(|0(\beta) \beta\rangle)=\sum_{\gamma}|\alpha(0) \alpha(\beta) \gamma\rangle \cdot\langle\gamma(\beta) \alpha|, \\
& E_{\tau} \rho(|\alpha(\beta) \gamma\rangle)= \pm\left|\tau(\alpha) \alpha^{\tau}(\beta) \gamma^{\tau}\right\rangle \cdot\left\langle\gamma^{\tau}(\gamma) \tau\right| \quad\left(\begin{array}{l}
- \text { if } \alpha=\beta=\gamma=\sigma \\
\text { totherwise }
\end{array}\right), \\
& E_{\sigma} \rho(|\tau(\tau) 0\rangle)=|\sigma(\tau) \sigma(\tau) \sigma\rangle \cdot\langle\sigma(0) \sigma|,
\end{aligned}
$$




$$
\begin{aligned}
E_{\alpha} \rho(|\sigma(\tau) \sigma\rangle) & =-\sum_{\alpha}\left|\sigma(\sigma) \alpha(\tau) \alpha^{\tau}\right\rangle \cdot\left\langle\alpha^{\tau}(\sigma) \sigma\right|, \\
E_{\sigma} \rho(|\tau(\sigma) \sigma\rangle) & =\sum_{\alpha}(-1)^{d_{\alpha}}|\sigma(\tau) \sigma(\sigma) \alpha\rangle \cdot\langle\alpha(\sigma) \sigma|, \\
E_{\sigma} \rho(|\sigma(\sigma) 0\rangle) & =\sum_{\alpha} \frac{1}{2} \sqrt{d_{\alpha}}|\sigma(\sigma) \alpha(\sigma) \sigma\rangle \cdot\langle\sigma(0) \sigma|, \\
E_{\sigma} \rho(|\sigma(\sigma) \tau\rangle) & =\sum_{\alpha}(-1)^{d_{x}} \frac{1}{2} \sqrt{d_{\alpha}}|\sigma(\sigma) \alpha(\sigma) \sigma\rangle \cdot\langle\sigma(\tau) \sigma|, \\
E_{\alpha} \rho(|\sigma(\sigma) \sigma\rangle) & =\sum_{\alpha \neq \sigma} \operatorname{sig}(\alpha)\left(|\sigma(\sigma) \sigma(\sigma) \alpha\rangle \cdot\left\langle\alpha(\sigma) \sigma\left|+\sqrt{\frac{1}{2}}\right| \sigma(\sigma) \alpha(\sigma) \sigma\right\rangle \cdot\langle\sigma(\sigma) \sigma|\right),
\end{aligned}
$$

where we put $[\alpha][\tau]=:\left[\alpha^{\tau}\right], \operatorname{sig}(0)=1, \operatorname{sig}(\tau)=-1$, and adopted a simplifying notation appropriate when there is only one channel of a given type, $N_{\alpha \beta}^{\gamma} \leqq 1$ :

$$
\begin{aligned}
T_{e_{1}} \cdots T_{e_{n}} & =T_{\eta}=\left|\alpha_{0}\left(\beta_{1}\right) \alpha_{1} \cdots \alpha_{n-1}\left(\beta_{n}\right) \alpha_{n}\right\rangle, \\
T_{e_{n}}^{*} \cdots T_{e_{1}}^{*} & =T_{\eta}^{*}=\left\langle\alpha_{n}\left(\beta_{n}\right) \alpha_{n-1} \cdots \alpha_{1}\left(\beta_{1}\right) \alpha_{0}\right|
\end{aligned}
$$

for $e_{i}$ of type $\left(\alpha_{i-1}, \beta_{i}, \alpha_{i}\right)$, displaying the successive transitions from the sector $\alpha_{i-1}$ to the sector $\alpha_{i}$ made by the charge $\beta_{i}$.

We shall first identify $u(g) \in \mathscr{M}$. For this purpose consider linear maps in $\left(\rho^{n+1} \mid \rho\right)$, defined on $T_{\eta}$ as follows. First split $\eta$ into three pieces (cf. (A.5)): the first piece is determined by $\alpha_{0}, \ldots, \alpha_{v-1}=\sigma$, the second one by $\alpha_{v}, \ldots, \alpha_{\mu} \neq \sigma$, and the remainder begins with $\alpha_{\mu+1}=\sigma$. This implies $\beta_{v}=\beta_{\mu+1}=\sigma$ while $\beta_{v+1}, \ldots, \beta_{\mu} \neq \sigma$. The lengths of the pieces can be $n \geqq \mu \geqq v \geqq 0$. Then define

$$
\begin{aligned}
&\left|\sigma\left(\beta_{1}\right) \sigma \cdots \sigma(\sigma) \alpha_{v}\left(\beta_{v+1}\right) \alpha_{v+1} \cdots \alpha_{\mu}(\sigma) \sigma \cdots\right\rangle \stackrel{f_{2}}{\longmapsto} e^{\operatorname{sig}\left(\alpha_{v}\right) 2 \pi i / 4} \cdot\left|\sigma\left(\beta_{1}\right) \sigma \cdots \sigma(\sigma) \alpha_{v}^{\tau}\left(\beta_{v+1}\right) \alpha_{v+1}^{\tau} \cdots \alpha_{\mu}^{\tau}(\sigma) \sigma \cdots\right\rangle \\
& \stackrel{f_{2}}{\longmapsto}\left[\prod_{j=v}^{\mu} e^{\operatorname{sig}\left(\alpha_{j}\right) 2 \pi i / 3}\right]^{\varepsilon_{v}} \cdot\left|\sigma\left(\beta_{1}\right) \sigma \cdots \sigma(\sigma) \alpha_{v}\left(\beta_{v+1}\right) \alpha_{v+1} \cdots \alpha_{\mu}(\sigma) \sigma \cdots\right\rangle,
\end{aligned}
$$

with $\varepsilon_{v}=0$ or 1 . The "exceptional" paths for which (A.6) is not defined, namely those with $\alpha_{0}, \ldots, \alpha_{n}=\sigma$, are taken into themselves by both $f_{i}$. We easily check $f_{2} \circ f_{2}=f_{3} \circ f_{3} \circ f_{3}=f_{2} \circ f_{3} \circ f_{2} \circ f_{3}=i d$, hence setting

$$
u_{n}\left(g_{i}\right)=\sum_{\eta} f_{i}\left(T_{\eta}\right) T_{\eta}^{*}, \quad i=2,3
$$

with $T_{\eta}$ running over the bases (A.5) of $\left(\rho^{n+1} \mid \rho\right)$, provides a non-trivial homomorphism $S_{3} \rightarrow \mathscr{U}(\mathscr{M})$. In fact, $u_{n}\left(g_{i}\right)$ depend on $n$, but due to the fact that the effect of $f_{i}$ essentially depends only on the beginning of the path $\eta$, one may convince oneself that they are Cauchy series, the limit points of which in the closure $\mathscr{M}$ are from now on denoted by $u_{i}=u\left(g_{i}\right)$. While $\operatorname{tr}\left(u_{2}\right)=0$ is obvious (to $\operatorname{tr}\left(u_{n}\left(g_{2}\right)\right)$ contribute only the $3^{n}$ exceptional paths with $\operatorname{tr}\left(T_{\eta} T_{\eta}^{*}\right)=2 \cdot 4^{-n-1}$ each), one has to carefully adjust the values of $\varepsilon_{v}=0,1$ to ensure $\operatorname{tr}\left(u_{3}\right)=0$. This solves (3.13).

Next, we verify that the isometries $b_{0}=W_{0} \in \mathscr{K}_{0}$ and $\tilde{b}_{3}=\sum_{\alpha}\left|\tau(\alpha) \alpha^{\tau}\right\rangle$ are orthogonal and complete bases of $\mathscr{K}_{0}$ respectively $\mathscr{K}_{\tau}$ in the sense of (3.11), and (using (A.4)) satisfy the multiplication law (3.12) as well as the transformation laws 
$b_{0}^{*} \rho\left(u_{i}\right) b_{0}=u_{i}$ and $\tilde{b}_{3}^{*} \rho\left(u_{2}\right) \tilde{b}_{3}=-u_{2}, \tilde{b}_{3}^{*} \rho\left(u_{3}\right) \tilde{b}_{3}=u_{3}^{-1}$. The appearance of $u_{3}^{-1}$ instead of $u_{3}$ on the right-hand side of the last equation can be corrected by replacing $b_{3}=i \rho\left(u_{2}\right) \tilde{b}_{3}$ without affecting the other equations, thus solving $(3.11-14)$ for all $I, J=0,3$.

Next, we shall determine $b_{ \pm} \in \mathscr{K}_{\sigma}$ which solve the transformation law (3.14) for $I, J= \pm$ :

$$
\rho\left(u_{i}\right) b_{J} u_{i}^{*}=\sum_{I} b_{I} \mathscr{D}_{I J}\left(g_{i}\right),
$$

as well as the expansions (3.12) for either $I$ or $J=0,3$ :

$$
\begin{array}{ll}
\tilde{T}_{s(\sigma)}^{*} \rho\left(b_{0}\right) b_{ \pm}=b_{ \pm}, & \tilde{T}_{t(\sigma)}^{*} \rho\left(b_{ \pm}\right) b_{0}=b_{ \pm}, \\
\tilde{T}_{l}^{*} \rho\left(b_{3}\right) b_{ \pm}= \pm b_{ \pm}, & \tilde{T}_{r}^{*} \rho\left(b_{ \pm}\right) b_{3}= \pm b_{ \pm} .
\end{array}
$$

(Here, $l$ is of type $(\tau, \sigma, \sigma)$ and $r$ of type $(\sigma, \tau, \sigma)$.) Note that the linear equations (A.8), (A.9) in $\mathscr{K}_{\sigma}$ are equivalent to (3.14), (3.12) provided (3.11) is satisfied. The first pair of Eqs. (A.9) holds trivially for every $k \in \mathscr{K}_{\sigma}$. For the other equations, consider the linear maps $\mathscr{K}_{\sigma} \rightarrow \mathscr{K}_{\sigma}$ corresponding to left and right action of $u_{i}$ and to left and right bimodule tensoring with $b_{3}$ :

$$
\begin{array}{lll}
l_{2}: k \mapsto \rho\left(u_{2}\right) k, & r_{2}: k \mapsto k u_{2}, \\
l_{3}: k \mapsto \rho\left(u_{3}\right) k, & r_{3}: k \mapsto k u_{3}, \\
l_{b}: k \mapsto \tilde{T}_{l}^{*} \rho\left(b_{3}\right) k, & r_{b}: k \mapsto \widetilde{T}_{r}^{*} \rho(k) b_{3} .
\end{array}
$$

From the previous knowledge one can verify that the "left" maps $l_{*}$ commute with the "right" maps $r_{*}$, and that

$$
l_{b} l_{2}=-l_{2} l_{b}, \quad l_{b} l_{3}=l_{3} l_{b}, \quad l_{b}^{2}=l_{2}^{2}=l_{3}^{3}=\left(l_{2} l_{3}\right)^{2}=i d
$$

hold as well as the same equations with the right maps. Then there are simultaneous eigenvectors of $l_{b}$ and $r_{b}$ with eigenvalue +1 and of $l_{3} r_{3}$ with eigenvalue $e^{2 \pi i / 3}$. Calling such an eigenvector $b_{+}$and putting $b_{-}=l_{2} r_{2}\left(b_{+}\right)$, yields (A.8) and (A.9) by virtue of (A.11), as well as

$$
b_{+}^{*} b_{-}=0, \quad b_{-}^{*} b_{-}=u_{2}\left(b_{+}^{*} b_{+}\right) u_{2}^{*},
$$

that is, $b_{-}$is an isometry iff $b_{+}$is an isometry. This would also ensure the completeness relation (3.11), since $b_{+} b_{+}^{*}+b_{-} b_{-}^{*}$ would be a projection in $\mathscr{M}$, majorized by $E_{\sigma}$ and having the same trace $d_{\sigma} / d=1 / 2$ as $E_{\sigma}$.

Although isometries in $\mathscr{K}_{\sigma}$ abound, we did not yet identify one explicitly in the required eigenspace for $b_{+}$. Apart from this (minor) problem, there remains the non-linear problem to solve (3.12) for $I$ and $J= \pm$. Certainly, more powerful mathematical techniques are necessary to prove our conjecture, and to proceed for the general case of braid group statistics. However, from our explicit calculations with the examples above we draw much confidence that the structures available from Theorem 2.7 are sufficiently rich for a systematic study of finite-dimensional (?) quantum symmetry by "thinning out the master symmetry."

We want to include a remark concerning the possibility of non-abelian ordinary group symmetry (and scalar Clebsch-Gordan expansions) associated with braid group statistics. The abelian case has been treated in Sect. 3.1. We note that for the superselection structure of the above $S_{3}$ theory, there exists another braided structure for the category of intertwiners; indeed each of the three statistics 
operators

$$
\begin{aligned}
\varepsilon(\rho, \rho)= & \text { (obvious terms) }+|\tau(\tau) 0\rangle\langle 0(\tau) \tau|+(|\tau(\sigma) \sigma\rangle\langle\sigma(\tau) \sigma|+\text { c.c }) \\
& +\omega^{-1}|\sigma(\sigma) 0\rangle\left\langle 0(\sigma) \sigma\left|-\omega^{-1}\right| \sigma(\sigma) \tau\right\rangle\langle\tau(\sigma) \sigma|+\omega| \sigma(\sigma) \sigma\rangle\langle\sigma(\sigma) \sigma|
\end{aligned}
$$

with $\omega_{\sigma}=\omega$ a third root of unity, is compatible with the axioms, given the endomorphism (A.4). The case $\omega=1$ is the above case of permutation group statistics, while the other two (which are conjugate to each other) are realized in appropriate (sub-)models of chiral algebras, e.g., the three sectors of integer isospin in an SU(2) Wess-Zumino-Witten model at level 4 . Then the same bases $b_{I}$ solve the conditions (3.11-14) with the same representatives $u$ of the gauge group, while only the numerical coefficients of the commutation relations (2.39) change. To be specific, at space-like distance the field components $\varphi_{0}$ and $\varphi_{3}$ commute among each other and with the doublet $\varphi_{ \pm}$, while for the latter if $\varphi$ is localized at the right of $\varphi^{\prime}$,

$$
\begin{aligned}
& \varphi_{ \pm} \varphi_{ \pm}^{\prime}=\omega \cdot \varphi_{ \pm}^{\prime} \varphi_{ \pm}, \\
& \varphi_{ \pm} \varphi_{\mp}^{\prime}=\omega^{-1} \cdot \varphi_{\mp}^{\prime} \varphi_{ \pm} .
\end{aligned}
$$

One might wonder how the two components of an irreducible doublet can have different commutation relations with a given other component, but the twodimensional representation (A.2) of $S_{3}$ perfectly respects (A.14). There is more reason to wonder about the anyonic appearance of (A.14). Indeed, it is known that upon including the local field $\varphi_{3}$ into the algebra of observables, the superselection structure changes into that of an anyonic $\mathbb{Z}_{3}$ theory, or, turning the argument around, the observables of the non-abelian $S_{3}$ theory are those observables of the $\mathbb{Z}_{3}$ theory which are invariant under an additional $\mathbb{Z}_{2}$ symmetry.

Acknowledgements. It is a pleasure to thank G. Mack and V. Schomerus for making their preprint [5] available to me in statu nascendi and for discussions about the apparent overlap of our scenarios, as well as $\mathrm{K}$. Fredenhagen for asking critical questions, and the Mathematical Sciences Research Institute in Berkeley for hospitality during a late stage of this work.

Note added in proof. I am indebted to R. Longo for clarifying discussions during a visit at the II. University of Rome, which resulted in the following view of the present construction. Let us abbreviate $\mathscr{B}:=\mathscr{A} \otimes \mathscr{M}, i:=i_{\mathscr{A}} \otimes i_{\mathscr{M}}, \sigma:=\rho \otimes \rho: \mathscr{B} \rightarrow \mathscr{B}, E:=\sum_{\alpha} E_{\alpha} \otimes E_{\bar{\alpha}} \in \sigma(\mathscr{B})^{\prime} \cap \mathscr{B}, \mathscr{G}:=E \mathscr{B} E$, and let $Z:=\zeta^{*} \in\left(\sigma^{2} \mid \sigma\right) \cap \mathscr{B}$ (cf. Proposition 2.6), satisfying $Z^{*} Z=\left(\sum_{\alpha} d_{\alpha}^{2}\right) \cdot E$ and $Z Z^{*}=\sigma\left(Z^{*}\right) Z=$ $Z^{*} \sigma(Z)$. Then

$$
i(\mathscr{B})=E \sigma(\mathscr{B}) E=Z^{*} \sigma^{2}(\mathscr{B}) Z \subset \mathscr{F}=Z^{*} \sigma(\mathscr{B}) Z \subset \mathscr{G}=Z^{*} \mathscr{B} Z .
$$

The inclusion $i(\mathscr{B}) \subset \mathscr{F}$ (studied in this article) has index $\sum_{\alpha} d_{\alpha}^{2}$ in analogy to the fixpoint inclusion w.r.t. a finite group, and depth $>2\left(=2\right.$ in the abelian case). Its Jones projection is $E_{J}=E_{0} \otimes E_{0} \in \mathscr{G}$, and $\left\langle\mathscr{F}, E_{J}\right\rangle=\mathscr{G}$. In fact, the inclusion $i(\mathscr{B}) \subset \mathscr{G}$ is equivalent to an endomorphism $\bigoplus_{\alpha} \rho_{\alpha} \otimes \rho_{\bar{\alpha}}$, the range of $E$ in $\sigma$ (cf. [23])

\section{References}

1. Doplicher, S., Roberts, J. E.: Compact Lie groups associated with endomorphisms of $C^{*}$-algebras. Bull. Am. Math. Soc. (New Series) 11, 333 (1984); Why there is a field algebra with a compact gauge group describing the superselection structure in particle physics. Commun. Math. Phys. 131, 51 (1990) 
2. Doplicher, S., Haag, R., Roberts, J. E.: Local observables and particle statistics. I + II. Commun. Math. Phys. 23, 199 (1971) and 35, 49 (1974)

3. Fredenhagen, K., Rehren, K.-H., Schroer, B.: Superselection sectors with braid group statistics and exchange algebras. I: General theory. Commun. Math. Phys. 125, 201 (1989)

4. Drinfel'd, V. G.: Quantum groups. In: Proceedings of the International Congress of Mathematicians (Berkeley 1986) Gleason, A. G. (ed.) New York: Academic Press 1987, p. 799; Quasi Hopf algebras and Knizhnik-Zamolodchikov equations. In: Problems of Modern Quantum Field Theory, Proceedings Alushta (USSR) 1989. Belavin, A. A. et al. (eds.) Berlin, Heidelberg, New York: Springer 1989

5. Mack, G., Schomerus, V.: Quasi Hopf quantum symmetry in quantum theory. Preprint DESY 91-037 (Univ. Hamburg 1991), to appear in Nucl. Phys. B

6. Fredenhagen, K.: Sum rules for spin in $(2+1)$-dimensional quantum field theory. In: Quantum Groups. Doebner, H. et al. (eds.) Lecture Notes in Physics vol. 370. Berlin, Heidelberg, New York: Springer 1990, p. 340

7. Tsuchiya, A., Kanie, Y.: Vertex operators in conformal field theory on $\mathbb{P}^{1}$ and monodromy representations of braid group. In: Conformal field theory and solvable lattice models. Adv. Stud. Pure Math. 16, 297 (1988); Rehren, K.-H., Schroer, B.: Einstein causality and Artin braids. Nucl. Phys. B312, 715 (1989)

8. Fröhlich, J.: Statistics of fields, the Yang-Baxter equation, and the theory of knots and links. In: Nonperturbative quantum field theory, Proceedings Cargèse 1987. G. 't Hooft et al. (eds.) London: Plenum Press 1988, p. 71

9. Roberts, J. E.: Lectures on algebraic quantum field theory. In: Algebraic theory of superselection sectors. Kastler, D. (ed.) Singapore: World Scientific 1990, pp. 1-112

10. Cuntz, J.: Simple $C^{*}$ algebras generated by isometries. Commun. Math. Phys. 57, 173 (1977)

11. Goodman, F. M., delaHarpe, P., Jones, V.: Coxeter Graphs and Towers of Algebras, MSRI Publ. (eds.) 14. Berlin, Heidelberg, New York: Springer 1989

12. Pimsner, M. V., Popa, S.: Entropy and index for subfactors. Ann. Sci. Éc. Norm. Sup. $4^{e}$ sér. 19, 57 (1986)

13. Ocneanu, A.: Quantized groups, string algebras, and Galois theory for algebras. In: Operator Algebras and Applications, Vol. 2, Evans, D. E. et al. (eds.) London Math. Soc. Lecture Notes Series vol. 135. Cambridge: Cambridge University Press 1988, p. 119

14. Jones, V. F. R.: Index for subfactors. Invent. Math. 72, 1 (1983)

15. Longo, R.: Index of subfactors and statistics of quantum fields. I+ II. Commun. Math. Phys. 126, 217 (1989) and 130, 285 (1990)

16. Doplicher, S.: Local observables and the structure of quantum field theory. In: Algebraic Theory of Superselection Sectors. Kastler, D. (ed.) Singapore: World Scientific 1990, p. 230; Doplicher, S., Roberts, J. E.: A new duality theory for compact groups. Invent. Math. 98, 157 (1989)

17. Reshetikhin, N. Yu., Turaev, V. G.: Ribbon graphs and their invariants derived from quantum groups. Commun. Math. Phys. 127, 1 (1990)

18. Moore, G., Seiberg, N.: Classical and quantum conformal field theory. Commun. Math. Phys. 123, 177 (1989)

19. Rehren, K.-H.: Space-time fields and exchange fields. Commun. Math. Phys. 132, 461 (1990)

20. Buchholz, D., Mack, G., Todorov, I.: The current algebra on the circle as a germ for local field theories. Proceedings Annecy 1988. Nucl. Phys. B (Proc. Suppl.) 5B, 20 (1988)

21. Rehren, K.-H.: Quantum symmetry associated with braid group statistics. In: Quantum Groups, Proceedings Clausthal-Zellerfeld 1989. Doebner, H. et al. (eds.) Lecture Notes in Physics vol. 370. Berlin, Heidelberg, New York: Springer 1990, p. 318

22. Witten, E.: Quantum field theory and the Jones polynomial. Commun. Math. Phys. 121, 351 (1989); Segal, G.: Two-dimensional conformal field theories and modular functors. In: Proceedings of the IX ${ }^{\text {th }}$ Intern. Congr. on Mathematical Physics (Swansea 1988), eds. B. Simon et al. (eds.) London: Adam Hilger 1989, p. 22

23. Rehren, K.-H.: Quantum symmetry associated with braid group statistics. II. To appear in: Quantum Groups II, proceedings of the II. Wigner Symposium, Goslar 1991, Doebner, H. et al. (eds.) 